\title{
Interference Mitigation Framework for Cellular Mobile Radio Networks
}

\author{
Wolfgang Mennerich, ${ }^{1}$ Michael Grieger, ${ }^{2}$ Wolfgang Zirwas, ${ }^{3}$ and Gerhard Fettweis ${ }^{2}$ \\ ${ }^{1}$ Focubeam GmbH, Germany \\ ${ }^{2}$ Vodafone Chair Mobile Communications Systems, TU Dresden, Germany \\ ${ }^{3}$ Nokia Siemens Networks, Germany
}

Correspondence should be addressed to Wolfgang Mennerich; wolfgang.mennerich@alumni.tu-berlin.de

Received 7 May 2012; Accepted 10 August 2012

Academic Editor: Sumei Sun

Copyright (C) 2013 Wolfgang Mennerich et al. This is an open access article distributed under the Creative Commons Attribution License, which permits unrestricted use, distribution, and reproduction in any medium, provided the original work is properly cited.

\begin{abstract}
For today's cellular mobile communication networks, the needed capacity is hard to realize without much more of (expensive) bandwidth. Thus new standards like LTE were developed. LTE advanced is in discussion as the successor of LTE and cooperative multipoint transmission (CoMP) is one of the hot topics to increase the system's capacity. System simulations often show only weak gains of the signal-to-interference ratio due to high interference from noncooperating cells in the downlink. This paper presents an interference mitigation framework to overcome the hardest issue, that is, the low penetration rate of mobile stations that can be served from a cluster composed of their strongest cells in the network. The results obtained from simulation tools are discussed with values resulting from testbed on the TU Dresden. They show that the theoretical ideas can be transferred into gains on real systems.
\end{abstract}

\section{Introduction}

From the beginning of mobile radio communication there is a constant effort to reduce intercell interference to an acceptable level. Since the success of the worldwide deployed Global System Mobile (GSM) [1] the enormous increased number of users together with over proportionally increasing traffic demands have put this topic into the main focus of research.

Wireless transmitters generally spread power over large areas, and at very low frequencies even worldwide communication is possible [2]. In current cellular deployments, carrier frequencies ranging from a few hundred $\mathrm{MHz}$ to several $\mathrm{GHz}$ are being used. At these frequency bands, the radiation of a single cell will typically cover smaller areas, but cell diameters of $100 \mathrm{~km}$ and more are still possible in rural areas. In urban areas intersite distance (ISD) of few $100 \mathrm{~m}$ to few $\mathrm{km}$ are quite common, leading to a so-called interference limited scenario, where the achievable performance is mainly bounded by the intercell interference (ICI) and not the receiver noise [3].

Interference power decreases with distance, which in the beginning of cellular communications has been the main means for overcoming intercell interference, leading to the well-known frequency reuse concept. Important enhancements were the introduction of frequency reuse 1 systems by code division multiple access (CDMA) in the so-called 3 rd generation of mobile systems. On the downside the performance of this scheme suffers from the near far effect and a variable soft capacity of the system [4].

In the standardization of the current 3GPP standard named LTE, CDMA was replaced in the downlink by orthogonal frequency division multiple access (OFDMA), being again a frequency reuse 1 system, but providing additionally multiuser scheduling gains by fast adaptation to frequency selective channel conditions [5]. This concept allows also fast adaptation to interference conditions and thereby approaches the performance of the so-called inter-cell interference coordination (ICIC) schemes. ICIC harmonizes resources usage between cell edge and cell centre users over neighbouring cells [6] depending on their mutual interference. Note ICIC has generated also a lot of interest under the name of fractional frequency partitioning (FFP) with a different power allocation for cell center and cell edge users on different frequency subbands. 
In long-term evolution (LTE) Release 10 and now 11 there is a lot of discussions about the so-called enhanced ICIC (eICIC) schemes in the context of heterogeneous networks comprising pico- and macro- or femto- and macro-stations. By transmitting the so-called almost blank subframes (ABS) - almost blank because the common reference signals (CRS) have to be transmitted continuously for backward compatibility - in time domain subframes with different level of interference are generated, providing some options for enhanced scheduling.

In 2000, Baier et al. [7] proposed the idea of cooperative transmission systems-also known as network multiple input multiple output (MIMO) or for example in 3rd generation partnership project (3GPP) as cooperative multi point transmission (CoMP). This is a much more fundamental solution, partly being inspired from the research in the area of MIMO systems. Many groups predicted large theoretical performance gains with spectral efficiencies being several factors higher than that of conventional mobile radio systems. For example for 2 and 4 antennas per cell (BS) and mobile station (MS) 10th of bits/s/Hz/cell have been promised at signal to noise and interference ratios (SINRs) of less than 10 to $20 \mathrm{~dB}$ [8-10].

Common to all these approaches is that a network wide precoder over all cells including all MSs is assumed, which eliminates any interference after proper precoding. In practical mobile radio systems, network wide precoding is unrealistic as networks might span a whole city or even a whole country. Neither an according backhaul network can be implemented, nor channel estimation as well as the reporting of all channel components is feasible. For that reason it is necessary to partition the network into so-called cooperation areas (CAs) connecting a limited number of cells. Unfortunately, this step turns the interference free system back into an interference limited one, because of the remaining intercooperation area interference. For quite sometime a lot of research concentrated on the area of clustering [11], applying different types of optimization algorithms to this nonconvex optimization problem.

Despite the research effort, there is still a significant gap between the theoretical predicted gains and the results observed from system level simulations so far [12]. One main activity in this area is the so-called 3GPP LTE CoMP study item, which started in LTE Release 10, has been re-invoked for LTE Release 11 and finally has been converted into a 3GPP LTE working item in September, 2011 [13]. While CoMP includes many different kinds of cooperation like coordinated scheduling (CS), coordinated beamforming (CB), dynamic or fast cell selection (DCS), and so forth, in our contribution the focus is mainly on joint precoding (JP) or more specifically on joint transmission (JT) CoMP. JT CoMP is generally accepted to be the scheme with the highest potential gains, as signals being interferers in noncooperative scenarios are turned into useful signal power for JT CoMP. This is the main benefit over pure interference avoidance strategies such as CS/CB. Reference [14] contains the best so far achieved results for the main $3 \mathrm{GPP}$ CoMP schemes. In case of JT CoMP the best performance for a $4 \times 2$ MIMO system doing joint transmission over 9 cells was $4.028 \mathrm{bit} / \mathrm{s} / \mathrm{Hz} / \mathrm{cell}$, being far away from the projected $10 \mathrm{bit} / \mathrm{s} / \mathrm{Hz} /$ cell from theoretical analysis.

So one main issue to be tackled in this contribution is to reveal and analyze a fundamental limit of practical and realizable CoMP schemes, mainly due to the required clustering and/or due to different assumptions for the radio channels. Note, for theoretical analysis Rayleigh channels are often assumed, which are known to have an optimal eigenvalue distribution, while macrocellular radio channels like the spatial channel model extended (SCME) as being used in 3GPP [15] may suffer from correlation of collocated antenna elements and certain line of sight (LOS)/nonline of sight (NLOS) probabilities.

The important question is whether the optimization problem is limited by fundamental reasons, for example, esteeming from clustering, or just due to the dimension of the optimization problem. The first case would be in combination with its significant complexity almost a killing point against JT CoMP, while in the second case it would be just a matter of doing things right.

We have no final answer to this question yet, but in this contribution we propose an overall framework for interference mitigation of which JT CoMP is just one of the building blocks. Based on an in depth analysis of interference conditions, scheduling effects, and so forth different concepts are combined to get much closer to the theoretical results of network wide cooperation with clustered cooperation areas in macrocellular radio environments.

Reference [16] mentions the importance of interference and provides an optimal power control provided for two opportunistic cooperative base stations. However the authors mention that the algorithm does not bring performance benefits if there are only cell edge users. Besides these ideas [17], suggests a concept to improve the bandwidth efficiency with two-way relay nodes where these nodes have multiple antennas. Upper bounds regarding symbol error probability are derived as well as the order of diversity of the two proposed schemes.

The proposed framework is more than CoMP and includes techniques like interference rejection combining (IRC), multiuser MIMO (MU-MIMO), interference floor shaping, wideband beamforming, antenna tilting, power adaptation, clustering, user grouping, effective precoding including $T x$ diversity, per cell and per cooperation area scheduling, optimum channel state information (CSI) estimation, and according to low rate but accurate feedback links.

Implementation of specific issues like the just mentioned CSI estimation and feedback as well as intersite synchronization, backhaul overhead, and so forth are investigated in more detail in the EU founded project Artist4G [18], while this contribution has its main focus on maximizing system level gains under ideal conditions. In the end, both parts have to be combined appropriately to form an overall system concept.

Some items like partial CoMP, cover shifts or interference floor shaping by cooperation wide vertical beam steering have been partly described in $[19,20]$, while the CoMP scheduler results and verification of main parts measurements in the real world CoMP testbed from TUD [21] are presented here for the first time. 
The main outcome of the overall proposed concept is a factor of 2.4 higher spectral efficiency than achieved so far by MU MIMO and about 1.8 times higher than for the up to date best 3GPP JT CoMP scheme as being given in [13]. This we think is really a great progress.

\section{Contribution of This Paper}

In the introduction, an overview about the issues relevant for interference mitigation based on JP CoMP was given. This chapter presents an interference mitigation framework designed to overcome the low penetration rate of MS that can be served by their individual set of network wide strongest BSs. To reach that goal, several concepts are combined to tackle each issue that hinders a higher penetration rate of MSs with high SIRs. These concepts can be roughly categorized into the following points.

(i) Oversized Clusters. Oversized clusters provide a higher probability that MSs find all of their strongest BSs in a single cluster.

(ii) Partial User Centric Assignment. It is sufficient if MS has only a subset of its serving BSs as its network wide strongest ones. This increases the probability to find multiple MSs to be served jointly from the same BS cluster.

(iii) Overlapping Clustering. The clusters are overlapping such that, around the border of two clusters, a third one is added to serve the cluster-edge-MSs as center MSs.

(iv) Interference Floor Shaping. Residual intercooperation area interference is reduced by a suitable combination of wideband (WB) beamforming and per WB beam individual vertical antenna tilting. This is done utilizing the multiple antenna elements (AEs) mounted at BSs.

\section{Considered Network and Signal Model}

We consider a downlink scenario within a cellular network with transmitting BSs and receiving MSs. The cells are organized in sites with three cells per site each covering a sector of $120^{\circ}$ as illustrated in Figure 2 consisting of three adjacent sites. Each BS covers a single cell. The mathematical notation is listed in Table 2.

First, a simple single input single outpout (SISO) radio channel between MS $m$ and BS $b$, each with only one AE, is introduced as complex scalar value $h_{m b}$. If MSs and BSs have multiple AEs, denoted by $N_{R X}$ and $N_{T X}$, respectively, the channel $h_{m b}$ becomes a matrix $\mathbf{H}_{m b} \in \mathbb{C}^{N_{R X} \times N_{T X}}$. All of these values represent the channel for a certain set of 12 subcarriers in the base band, accordingly physical resource block (PRB) in case of LTE. Radio channels from multiple BSs, denoted by a set, for example, $\mathscr{B}=\left\{b_{1}, \ldots, b_{B}\right\}$, are concatenated horizontally. Similar, the radio channels to multiple MSs in a set (e.g., $\left.\mathscr{M}=\left\{m_{1}, \ldots, m_{M}\right\}\right)$ are concatenated vertically. This and the concatenation to a matrix $\mathbf{H}_{\mathscr{M} \mathscr{B}}$ is shown in

$$
\begin{aligned}
\mathscr{B} & =\left\{b_{1}, \ldots, b_{B}\right\} \\
\mathscr{M} & =\left\{m_{1}, \ldots m_{M}\right\} \\
\Longrightarrow & {\left[\begin{array}{c}
\mathbf{H}_{m_{1} \mathscr{B}} \\
\vdots \\
\mathbf{H}_{m_{M} \mathscr{B}}
\end{array}\right]=\left[\mathbf{H}_{\mathscr{M} b_{1}} \cdots \mathbf{H}_{\mathscr{M} b_{B}}\right] . }
\end{aligned}
$$

3.1. Joint Precoding/Transmission. In the following, $\mathscr{M}_{b}$ is the set of MSs that are jointly served by BS $b$. We assume further that MSs have only one receive antenna, that is $N_{R X}=1$. Thus, $\mathbf{H}_{m b}=\mathbf{h}_{m b} \in \mathbb{C}^{1 N_{T X}}=\left[\mathbf{h}_{m_{b}}[1] \cdots \mathbf{h}_{m b}\left[N_{T X}\right]\right]$. To separate all jointly served MSs in the set $\mathscr{M}_{b}$, BS $b$ uses a precoding vector $\mathbf{v}_{m} \in \mathbb{C}^{N_{T X} \times 1}=\left[\mathbf{v}_{m}[1] \cdots \mathbf{v}_{m}\left[N_{T X}\right]\right]^{T}$ for each MS $m \in \mathscr{M}_{b}$. The precoding vectors are columns from the joint precoding matrix $\mathbf{V}_{b}$, used at $\mathrm{BS} b$. It is calculated based on the channel matrix $\mathbf{H}_{\mathscr{M}_{b} b}$ and could be, for example, the moore penrose pseudo-inverse of $\mathbf{H}_{\mathscr{M}_{b} b}$, denoted by $\mathbf{H}_{\mathscr{M}_{b} b}^{\dagger}$.

Intracell interference received at MS $m$ is caused by all other data streams transmitted by BS $b$ for MSs $k$ with $k \in$ $\mathscr{M}_{b} \backslash m$. Finally, an additive white and Gaussian distributed noise signal $n$ with power $\sigma_{n}^{2}$ is assumed.

If MS $m$ is served just from BS $b$ that serves all MSs in set $\mathscr{M}_{b}$, the signal $y_{m}$ received at MS $m$ can be expressed by

$$
y_{m}=\mathbf{h}_{m b} \mathbf{v}_{m} d_{m}+\sum_{k \in M_{b} \backslash m} \mathbf{h}_{m b} \mathbf{v}_{k} d_{k}+\stackrel{\vee}{I}_{m}+n
$$

with $d_{m}$ being the transmitted data symbol for MS $m$ and interference $I_{m}$ from other cells. If the transmit power is denoted by $p_{m}=\left|d_{m}\right|^{2}$, the wanted signal power $\widehat{S}(m, b)$, intra cell interference $\widehat{I}(m, b)$, and the interference $I(m, q)$ from another BSs $q$ at MS $m$ are given with

$$
\begin{gathered}
\widehat{S}(m, b)=p_{m}\left\|\mathbf{h}_{m b} \mathbf{v}_{m}\right\|_{2}^{2}, \\
\widehat{I}(m, b):=\sum_{k \in \mathscr{M}_{b} \backslash m} p_{k}\left\|\mathbf{h}_{m b} \mathbf{v}_{k}\right\|_{2}^{2}, \\
I(m, q):=\sum_{k \in M_{q}} p_{k}\left\|\mathbf{h}_{m q} \mathbf{v}_{k}\right\|_{2}^{2} \quad q \neq b .
\end{gathered}
$$

Interference between these MSs mostly depends on the precoding algorithm, like for example, MMSE or zero forcing. These algorithms strive to suppress interference power below the noise or in the case of zero forcing to remove it completely. In reality an interference floor remains, due to impairments such as channel estimation errors and inter-carrier interference. The error floor depends also on the precoding algorithm's "robustness." For instance, the zero forcing algorithm is quite sensitive against precoding errors and more robust solutions are available $[22,23]$, especially for multiple user separation $[24,25]$. We focus on methodical gains achieved by cooperation between multiple, distributed BSs and not on the joint precoding itself. Thus, we assume that joint precoding algorithms, channel prediction and estimation, and so forth are already applied in an optimal manner [26-28]. 
3.2. Precoding Normalization Loss. Data streams of all MSs $\mathscr{M}_{b}$ served by BS $b$ are transmitted in parallel. The transmit power used at that $\mathrm{BS}$ is limited by $P_{\max }^{\mathrm{BS}}$, yielding to a sum power constraint for all signals transmitted in parallel. Furthermore, each single AE at the BS has also a maximum transmit power given by $P_{\max }^{\mathrm{AE}}$. Hence, we have a second sum power constraint. These power limitations can either be considered within the joint precoding algorithm as presented, for example, in $[29,30]$ or by an additional normalization between all jointly transmitted data streams. Hence, the precoding vectors, like $\mathbf{v}_{m}^{\prime}$ in this article, are normalized. The application of a separated precoding and normalization is typically suboptimal and results in reduced SINR. This precoding normalization loss (PNL) is denoted by $\alpha(m, b)$ :

$$
\begin{gathered}
\mathbf{v}_{m} \| \mathbf{v}_{m}^{\prime} \quad \forall m \\
P_{\max }^{\mathrm{AE}} \geqslant \sum_{m \in \mathscr{M}_{b}} p_{k}\left|\mathbf{v}_{m}^{\prime}[k]\right|^{2} \quad \forall k \\
P_{\max }^{\mathrm{BS}} \geqslant \sum_{m \in \mathscr{M}_{b}} p_{k}\left\|\mathbf{v}_{m}^{\prime}\right\|_{2}^{2} \quad \forall b \\
\Longrightarrow \alpha(m, b):=\frac{\left\|\mathbf{h}_{m b} \mathbf{v}_{m}^{\prime}\right\|_{2}^{2}}{\left\|\mathbf{h}_{m b} \mathbf{v}_{m}\right\|_{2}^{2}}
\end{gathered}
$$

3.3. Signal-to-Interference Ratio. This article is about interference mitigation. Thus, we assume an interference limited system with noise power $\sigma_{n}^{2}$ being negligible compared to the interference power. Hence, the signal to interference ratio (SIR) is used in the following. The SIR seen at MS $m$ which is served by BS $b$ can be clearly described using the aforementioned notation:

$$
\operatorname{SIR}(m, b)=\frac{\alpha(m, b) \widehat{S}(m, b)}{\widehat{I}(m, b)+\sum_{q \neq b} I(m, q)} .
$$

3.4. Cooperation between Base Stations. A BS cluster is described by a set $\mathscr{B}=\left\{b_{1}, \ldots, b_{\Omega}\right\}$ of $\Omega$ cooperating BSs with cardinality $|\mathscr{B}|=\Omega$. The set $\mathscr{M}_{\mathscr{B}}=\left\{m_{1}, \ldots, m_{M}\right\}$ of $M$ MSs is served jointly by all BSs in $\mathscr{B}$. Equivalently as $\mathscr{M}_{b}$ is the set of all MSs served by BS $b, \mathscr{M}_{\mathscr{B}}$ is defined as the union of all served MSs from the cooperating BSs in $\mathscr{B}$ :

$$
\mathscr{M}_{\mathscr{B}}=\bigcup_{b \in \mathscr{B}} \mathscr{M}_{b}
$$

The SIR defined in (5) is now extended:

$$
\operatorname{SIR}(m, \mathscr{B}) \approx \frac{\alpha(m, \mathscr{B}) \widehat{S}(m, \mathscr{B})}{I(m, \mathscr{B})+\sum_{q \notin \mathscr{B}} I(m, q)} .
$$

Assuming that a certain MS $m$ is assigned to BS $b_{1}$, the BS indices $b_{1}, b_{2}, \ldots$ fulfil a special sorting of the BSs.

(i) The BS to which MS $m$ would be connected without any cooperation is denoted by $b_{1}$. Typically this is the $\mathrm{BS}$ with the strongest radio channel for MS $m$. (ii) The set of all BSs at the same site $\mathscr{B}^{\text {site }}$ with BS $b_{1}$ being one of them is denoted by $\mathscr{B}_{1}^{\text {site }}$, consisting of BSs $\left\{b_{1}, b_{2}, b_{3}\right\}$. Note that BS $b_{1}$ is connected to MS $m$ because it has the strongest radio channel to it, but BSs $b_{2}, b_{3}$ have in general an arbitrary connectivity to MS $m$, since they are only connected to MS $m$ now because they are located at the same site as BS $b$.

(iii) A CA is a special cluster consisting of three adjacent sites, that is, $\mathscr{B}_{1}^{\text {site }}, \mathscr{B}_{2}^{\text {site }}, \mathscr{B}_{3}^{\text {site }}$ like illustrated in Figure 2. Thus, the comprehensive set $\mathscr{C}$ contains 9 BSs $\left\{b_{1}, \ldots, b_{9}\right\}$. All BSs $b_{n}$ with $n>9$ and sites $\mathscr{B}_{k}^{\text {site }}$ with $k>3$ are not in that CA and cause interference at MS $m$. Note that, in general, $\mathscr{B}_{2}^{\text {site }}$ and $\mathscr{B}_{3}^{\text {site }}$, that is $\left\{b_{4}, b_{5}, b_{6}\right\}$ and $\left\{b_{7}, b_{8}, b_{9}\right\}$ have also arbitrary channel conditions as already explained above for $\mathrm{BS} b_{2}, b_{3}$.

Using that numbering scheme, the SIR for intra- and intersite cooperation is given by

$$
\begin{aligned}
\operatorname{SIR}\left(m,\left\{\mathscr{B}_{1}^{\text {site }}, \mathscr{B}_{2}^{\text {site }}, \mathscr{B}_{3}^{\text {site }}\right\}\right) & =\operatorname{SIR}(m, \mathscr{C}) \\
& \approx \frac{\alpha(m, \mathscr{C}) \widehat{S}(m, \mathscr{C})}{\widehat{I}(m, \mathscr{C})+\sum_{q \notin \mathscr{C}} I(m, q)} .
\end{aligned}
$$

\section{The Interference Mitigation Framework}

In general, cooperation between different BSs can be done in different ways. In this article, it is assumed that JP between BSs in a CA separates all jointly served MSs in the spatial domain, using the AEs of all cooperating BSs as one single and distributed antenna array. The precoding itself can be done in a so-called central unit (CU) like the one sketched in Figure 9 with $N_{T X}=2$ AEs for each of the three BSs in the cluster.

Now, we introduce the interference mitigation framework step by step. The specific parameter settings given below are an example for the explanation of the main concepts. The values like the size of the CAs, and so forth have been chosen based on practical considerations, but might be further optimized or adapted in case of other scenarios.

4.1. User Centric Assignment. For high SIRs, the clustering should be organized such that a cluster contains the network wide $\omega$ strongest BSs for each of its served MSs. This individual list of network wide strongest BSs is referred to as an MS's "wish list" in the following. The assignment is called "user centric" because it depends on the users, that is the MSs and their wish lists. In contrast, the assignment is network centric if cooperation areas are predefined on network level without adaptation to any wish lists. For user centric assignment the interference from adjacent clusters is weak as the strongest BSs belong to the serving cluster and all other BSs have lower power than the serving BSs. In realistic scenarios, user centric assignment is hard to realize because the BSs according to an MS's wish list often do not belong to a single predefined cluster and vary between different MSs. Since in scenarios with intrasite cooperation only BSs on the same site can cooperate, MSs will often have strong BSs at adjacent sites that cause strong inter site interference. Above all, this motivates the 
introduction of the aforementioned CAs because then, more of the strongest BSs might be in a single cluster, that is a CA.

Regarding the SIR expressions as expressed in (8) the goal of user centric assignments is to minimize the right side of the denominator by maximizing the connectivity $\left\|\mathbf{h}_{m \mathscr{B}}\right\|$ of an MS's serving cluster $\mathscr{B}$ :

$$
\left\{b_{1}, \ldots, b_{\Omega}\right\} \arg \max _{\mathscr{B}:|\mathscr{B}|=\Omega}\left\|\mathbf{h}_{m \mathscr{B}}\right\|_{2}^{2} .
$$

Within the optimization in (9), the number $\omega$ of requested strongest BS in the serving cluster is equal to the total amount $\Omega$ of BSs in a cluster. If the argument of the max-function is-by chance-identical to the $n$ 'th site $\mathscr{B}_{n}^{\text {site }}$, intrasite cooperation fulfils these requirements for user centric assignment with $\omega=3$, but often the strongest $\omega$ BSs are not collected at a single site. In that case, for example, two sites with 6 BSs must cooperate to have at least the strongest $\omega$ BSs in the serving CA. This case is referred to as partial user centric assignment and formulated in

$$
\left\{b_{1}, \ldots, b_{\Omega}\right\} \ni \arg \max _{\mathscr{B}:|\mathscr{B}|=\omega \leqslant \Omega}\left\|\mathbf{h}_{m \mathscr{B}}\right\|_{2}^{2} .
$$

However, the strongest BSs are often distributed over a larger area than just between two adjacent sites within a single CA. This illustrates the challenge of (partial) user centric assignment. Recalling the indexing introduced in Section 3.4, there is the following "user centric condition" concerning the connectivity:

$$
\left\|\mathbf{h}_{m, b_{n}}\right\| \geqslant\left\|\mathbf{h}_{m, b_{k}}\right\| \quad n \leqslant \omega<k .
$$

Equation (10) fulfils the user centric condition (11). With $\omega=\Omega$, also (9) does so. In the following, the latter case with $\omega=\Omega$ is referred to as "full user centric assignment" and $\omega<\Omega$ is called "partial user centric assignment." Due to the allocation of the strongest $\omega$ BS, the SIR depends not only on the cardinality of $\mathscr{B}$. For example, recall the indexing from Section 3.4: $b_{1}, \ldots, b_{3}$ belongs to the site cluster $\mathscr{B}_{1}^{\text {site }}$ while $b_{4}, \ldots, b_{6}$ and $b_{7}, \ldots, b_{9}$ belong to $\mathscr{B}_{2}^{\text {site }}$ and $\mathscr{B}_{3}^{\text {site }}$, respectively. Furthermore it is assumed that the connectivity from BSs that are not members of $\left\{b_{1}, \ldots, b_{9}\right\}$ is not relevant, that is the interference floor from BSs that are located further away from MS $m$. Now, we compare the SIRs resulting from two different serving clusters for MS $m$ under some further assumptions:

$$
\begin{gathered}
\left\|\mathbf{h}_{m, b_{n}}\right\| \geqslant\left\|\mathbf{h}_{m, b_{k}}\right\| \quad n \leqslant 3<k \\
\left\|\mathbf{h}_{m, b_{n}}\right\| \gg \mathbf{h}_{m, b_{k}} \| \quad n \leqslant 9<k \\
\left\|\mathbf{h}_{m, b_{n}}\right\| \approx\left\|\mathbf{h}_{m, b_{k}}\right\| \quad n, k \in\{4, \ldots, 9\} \\
\Longrightarrow \operatorname{SIR}\left(m, \mathscr{B}_{1}^{\text {site }}\right)<\operatorname{SIR}\left(m,\left\{\mathscr{B}_{1}^{\text {site }}, \mathscr{B}_{2}^{\text {site }}\right\}\right) \\
\operatorname{SIR}\left(m,\left\{\mathscr{B}_{1}^{\text {site }}, \mathscr{B}_{2}^{\text {site }}\right\}\right) \approx \operatorname{SIR}\left(m,\left\{\mathscr{B}_{1}^{\text {site }}, \mathscr{B}_{3}^{\text {site }}\right\}\right) \\
\operatorname{SIR}\left(m,\left\{\mathscr{B}_{1}^{\text {site }}, \mathscr{B}_{n}^{\text {site }}\right\}\right) \ll \operatorname{SIR}\left(m,\left\{\mathscr{B}_{1}^{\text {site }}, \mathscr{B}_{2}^{\text {site }}, \mathscr{B}_{3}^{\text {site }}\right\}\right) \\
n \in\{2,3\} .
\end{gathered}
$$

The example above makes clear that large cooperation gains can only be realized if all of the relevant interferers are in the serving cluster and contribute to the wanted signal for an MS, that is, the signal gain alone will not result in strong SIR gains. Thus, the most important issue is to combine the relevant interferers, that is the network wide strongest BSs into the serving cluster for each MS. The SIR can be increased more by interference mitigation of all relevant interferers than due to a signal gain. In the following, three concepts are presented that exploit these issues.

(i) Oversized Clusters. Partial user centric assignment is explicitly permitted. The conditions for user centric assignment are reduced to $\omega<\Omega$. The MSs should find now their $\omega$ strongest BSs within a single cluster of size $\Omega$. This is the notion behind the introduction of CAs because in their central area, MSs are surrounded with cooperating BSs.

(ii) Partial Channel Reporting. To realize such large clusters, only channels of the $\omega$ strongest BSs are reported from the MSs and used as transmitters for the desired signal. The channel coefficients to all unreported BSs are assumed to be zero. This concept is referred to as "partial channel reporting (PCR)" in the following.

(iii) Overlapping Clusters. For MSs positioned on the border between two CAs, a third (overlapping) CA with its center area at that border is added. Since it operates on orthogonal resources, for example, another frequency sub-band, interference towards the first two CAs is completely avoided. This overlapping scenario is illustrated in Figure 7.

4.2. Oversized Clustering. Note that PCR will introduce intraCA interference at signal power level as it would also appear from adjacent and strong BSs that do not belong to the serving CA, but for an MS, it is unimportant whether interference comes from an unreported BS inside of its serving cluster or any other BSs in the network. The advantage lies in the relaxed condition that only $\omega$ out of $\Omega$ BSs must be the strongest ones for the MS. This increases the so-called "penetration rate," that is, the percentage of MSs that can be served that way. Note that the penetration rate also depends on the probability to find enough MSs with identical wish lists, an issue which is addressed later.

One reason for the very low probability that MSs can be served in a full user centric assignment is the huge number of possibilities for wish lists of each single MS. Considering a network of $N_{\mathrm{BS}}$ BSs, the number of possibilities to select a group consisting of $\Omega$ BSs is given by the binomial coefficient:

$$
\left(\begin{array}{c}
N_{\mathrm{BS}} \\
\Omega
\end{array}\right)=\frac{N_{\mathrm{BS}} !}{\Omega !\left(N_{\mathrm{BS}}-\Omega\right) !} .
$$

Neglecting pathloss and assuming an equal distribution of the connectivity between each BS and MS, the probability that a certain group of $\Omega$ BSs is the optimum serving set of an arbitrary MS can be estimated just by the inverted binomial coefficient $\left(\begin{array}{c}N_{\mathrm{BS}} \\ \Omega\end{array}\right)^{-1}$. 
TABLE 1: Simulation parameter.

\begin{tabular}{lc}
\hline Parameter & Value \\
\hline Number of cells (BSs) & 57 \\
Number of sites & 19 \\
Cells (BSs) per cellsite & 3 \\
Sector width & $120^{\circ}$ \\
Physical AEs per BS & 4 \\
WB beams per BS & 2 \\
Hrz. WB beam direction & $\pm 30^{\circ}$ from broadside \\
Vrt. WB beam direction & $10^{\circ} / 15^{\circ}$ center/edge \\
Power adapt. WB beams & $0 /-6 \mathrm{~dB}$ center/edge \\
Number of subcarriers & 32 \\
Bandwidth per subcarrier & $180 \mathrm{kHz}$ \\
TxAEs & 4 \\
RxAEs & 1 \\
Joint precoding & Zeroforcing \\
Channelmodell & SCME \\
ISD & $500 \mathrm{~m}$ \\
Antenna tilting & $10^{\circ}$ and $15^{\circ}$ \\
Penetration loss & No \\
CSI & Ideal \\
\hline
\end{tabular}

TABLE 2: Mathematical notation.

\begin{tabular}{lc}
\hline Meaning & Notation \\
\hline Scalars, functions & $a$ \\
Vectors, matrix elements & $\mathbf{a}$ \\
Element $k$ of vector $\mathbf{a}_{h}$ & $\mathbf{a}_{n}[k]$ \\
Matrices & $\mathbf{A}$ \\
Sets & $\mathscr{A}$ \\
Cardinality of $\mathscr{A}$ & $|\mathscr{A}|$ \\
$|\mathscr{A}|=N$ & $\mathscr{A}^{N}$ \\
Vector a transposed & $\mathbf{a}^{T}$ \\
Vector a hermitian & $\mathbf{a}^{H}$ \\
Pseudo-inverse of matrix A & $\mathbf{A}^{\dagger}$ \\
\hline
\end{tabular}

Thus, the probability that an arbitrary set of BSs is selected and belongs to any of $N_{\mathscr{C}}$ clusters, that is, the penetration rate, is given by $N_{\mathscr{C}}\left(\begin{array}{c}N_{\mathrm{BS}} \\ \Omega\end{array}\right)^{-1}$. The penetration rate is increased using the relaxed assigning condition $\omega<$ $\Omega$ according to the PCR concept. Thus, there are $\left(\begin{array}{c}\Omega \\ \omega\end{array}\right)$ possibilities that an MS has its strongest $\omega$ BSs within a cluster consisting of $\Omega$ BSs. Furthermore, the fact that in general multiple MSs with an identical wish list are needed to form a group of jointly served MSs would drastically reduce the penetration rate, but the aforementioned relaxed condition $\omega<$ $\Omega$ allows grouping MSs with different wish lists since only their $\omega$ strongest BSs must be in the serving cluster of size $\Omega$.

Figure 3 contains results from a system level simulation (details in Table 1) and shows the penetration rate of MSs with 3, 6, and 9 of their strongest BSs in their serving CA. A significant increased penetration rate can already be expected if MSs should have their strongest 6 BSs in a single CA. If $\omega$ is decreased further to 3 , in combination with CAs of cardinality $\Omega=9$, we have penetration rates of more than $90 \%$.

4.3. Overlapping Clustering. If each possible cluster would be realizable, we have $N_{\mathscr{C}} \rightarrow\left(\begin{array}{c}N_{\mathrm{BS}} \\ \Omega\end{array}\right)$ and the penetration rate approaches one, but with a nonoverlapping clustering, the number of clusters that can be defined in parallel from $N_{\text {BS }}$ BSs is limited to $N_{\text {BS }} / \Omega$. Thus, overlapping clusters allow a higher probability that the wish list of a certain MS is identical to one of these clusters. On a first glance, the idea of overlapping clustering seems similar to fractional frequency partitioning, but the overlapping clustering is chosen such that each BS contributes to multiple CAs that overlap each other. The frequency subbands are assigned to these overlapping clusters such that each BS offers the complete system bandwidth distributed between all clusters it is contributing to. Thus, the MSs must be divided into subgroups served only by a fraction of the system's bandwidth. If these subbands are chosen big enough that each MS can use as much subcarriers as needed for its purposes, the MSs will not suffer from any bandwidth limitation. Of course, the multiplexing gain might be reduced by the reduced bandwidth here.

The overlapping clustering consists of different clustering layers, so called "cover shifts" built from a basic clustering that consists of nonoverlapping CAs as illustrated in Figure 2. Each of these clustering layers is "shifted" into different directions to be located directly on the borders between two adjacent CAs from the basic clustering. As aforementioned, overlapping clusters use different frequency subbands to avoid interference between different cover shifts. The shape of a CA according to Figure 2 allows to built 6 of such cover shifts as illustrated in Figure 4 from left to right. The triangles have their edges on the three sites forming a CA. The center area of each CA is highlighted within the triangles. From left to the right, Figure 4 depicts the basic clustering with more and more cover shifts being added to fill all gaps between the CAs of the basic clustering. With all six cover shifts, the overlapping is such that each border between two CAs in, for example, cover shift " $\mathrm{A}$ " is the centre area of another cover shift "B" as illustrated in Figure 7.

4.4. Partial Channel Reporting. As aforementioned, the high cooperation overhead might become an issue in CAs due to intersite cooperation. For full channel knowledge within a cluster, each channel coefficient on each subcarrier for each served MS must be known at each of the cooperating BSs. Furthermore, signals to be transmitted jointly according to Figure 9 must first be distributed between cooperating sites. The PCR concept reduces this overhead significantly, but since the full channel matrix between all transmit and receive antennas is needed for JP, the CU assumes all unreported radio channel coefficients simply to be zero.

A channel coefficient of zero means that no power can be transmitted. Thus, the joint precoding is calculated with wrong assumptions and MSs will see unknown interference from all unreported BSs.

As an example, we consider a channel matrix $\mathbf{H}_{\mathscr{M}_{\mathscr{B}} \mathscr{B}}$ between a cluster $\mathscr{B}=\{1, \ldots, 5\}$ and the jointly served MSs in $\mathscr{M}_{\mathscr{B}}=\{1, \ldots, 5\}$. In addition, we assume one $\mathrm{AE}$ 
at each BS and MS and that the first MS has only reported the channels corresponding to BSs 1,2, and 5. Thus, the resulting precoding matrix $\mathbf{V}_{\mathscr{B}}$ contains errors such that the underlined entries (corresponding to the AEs from unreported BSs) contain interference for the first MS:

$$
\begin{aligned}
& \mathbf{H}_{\mathscr{M}_{\mathscr{B}} \mathscr{B}}=\left(\begin{array}{lllll}
h_{11} & h_{12} & \frac{h_{13}}{h_{21}} & \frac{h_{14}}{h_{15}} & h_{15} \\
h_{21} & h_{22} & h_{23} & h_{24} & h_{25} \\
h_{31} & h_{32} & h_{33} & h_{34} & h_{35} \\
h_{41} & h_{42} & h_{43} & h_{44} & h_{45} \\
h_{51} & h_{52} & h_{53} & h_{54} & h_{55}
\end{array}\right)
\end{aligned}
$$

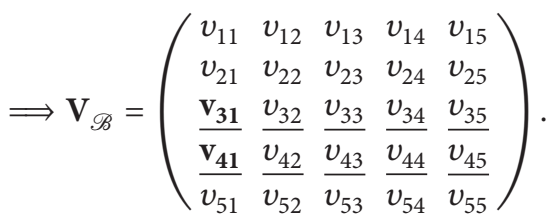

Since this kind of interference appears also in the data stream for the first MS, that is, the first column in V, the PCR concept bounds the achievable SIR, but in noncooperative scenarios the unreported BSs would be interferers as well. Thus, the SIR bound is not really limiting the capacity in real world scenarios.

It is important that each BS in the CA is reported by at least one MS to make sure that the channel matrix does not contain any rows which are completely zero. This can be guaranteed if the scheduling applies a simple rule.

(i) The set $\mathscr{M}_{\mathscr{B}}$ is composed such that each BS in $\mathscr{B}$ has at least one MS of which it is the strongest BS in $\mathscr{B}$.

(ii) Each MS reports at least its strongest BS in the CA.

This is easy to fulfil because MSs are generally connected to their strongest BS in the network. If each BS contributing to $\mathscr{B}$ adds one of "its" MSs to $\mathscr{M}_{\mathscr{B}}$, the wish lists from this set of MSs will span all BSs in $\mathscr{B}$. To increase the system's capacity, also more than one MS per BS can be served. This is of course limited by the number of AEs locally mounted at BSs.

There are a lot of different approaches how an MS can select the BS whose radio channels are reported. In this article, two of them are investigated: a "top- $N$ " and a threshold-based selection.

(i) A top $N$ selection means to select the strongest $N=\omega$ BSs in the serving cluster to be reported.

(ii) For threshold-based selection, the MS reports all BSs of the serving CA that have a radio channel connectivity stronger than a certain threshold below the strongest BS.

While Top $N$ selection yields to a variable SIR gain with a fixed cooperation overhead, the threshold-based selection has a variable cooperation overhead, but tries to keep a certain cooperation gain which is only limited by inter-CA interference.

With the same simulations as used for Figure 3 (see Table 1 for details) PCR in combination with an overlapping clustering according to Figure 4 has been simulated. Especially for MSs with a low SINR, the gains due to partial CoMP are significant and higher than those of intrasite cooperation.
4.5. Scheduling between Cover Shifts. The division into cover shifts with frequency subbands motivates a load balancing between them and the presented clustering in combination with PCR allows a certain degree of freedom there, because the scheduling of MSs between cover shifts can also be reduced to the reported $B S$.

With $\omega=3$ and the top- $N$ selection approach, the reported BSs can be distributed between one, two, or three different sites. If all of the reported BSs belong to the same site, the corresponding MS can be scheduled to each cover shift because the overlapping clustering as illustrated in Figure 4 was designed such that each site contributes to a CA in each cover shift. If the three strongest BSs are distributed between two sites, there are still two cover shifts left where these MSs could be scheduled to. The cover shift is only fixed if all three reported BSs belong to different sites. Figure 8 sketches the possible cover shifts for different locations of an MS's three network wide strongest BSs, denoted by the three dark hexagons. Of course, the number of sites that are included in a MS's set of reported BSs depends also on $\omega$. Until now, $\omega=3$ with a top- $N$ selection approach was assumed, but if the selection is done threshold based, $\omega$ only limited by $\Omega$.

Figure 6 compares the top- $N$ and threshold-based selection approaches. It comes out that a threshold of about $10 \mathrm{~dB}$ results in intrasite cooperation for $80 \%$ of all MS. Vice versa this means that the SIR is limited to $10 \mathrm{~dB}$ for $80 \%$ of all MSs due to intersite interference. This coincides with the low gains that are often seen in such simulations as already mentioned in the introduction of this article. The first idea for load balancing is to schedule MSs that can be scheduled only to one single cover shift first. Second, MSs with two "allowed" cover shifts are distributed between the different cover shifts with the goal in mind to equalize the number of MSs in each of these cover shifts. In a third step, all MSs that are free in their choice are added. As long as there are enough of the latter type of MSs to schedule, an equal load between all cover shifts can be reached. This technique is described more in detail in [31]. More powerful scheduling ideas are sketched briefly later in this article.

4.6. Interference Floor Shaping. Both, overlapping clusters and PCR exploit the fact that the main cooperation gain arises from a significant interference reduction and less from a signal gain. Despite oversized and overlapping clusters there is still a considerable amount of inter-CA interference, especially for CA edge users. In the following, we focus on the mitigation of that interference part.

Multiple antennas at BSs can be used for a static wideband beamforming before joint preprocessing is applied. This allows steering the transmit power more towards the center of a CA for mitigating inter-CA interference. The concept is presented in Figure 1. The depicted beams are divided into the so-called "centre beams" and "edge-beams." Each BS has for example, 4 AEs and uses them to provide 2 wideband beams. Additionally to the horizontally beamforming, edge beams have a stronger down tilt than centre beams and are reduced in their transmit power to further mitigate the interference power to adjacent CAs. 


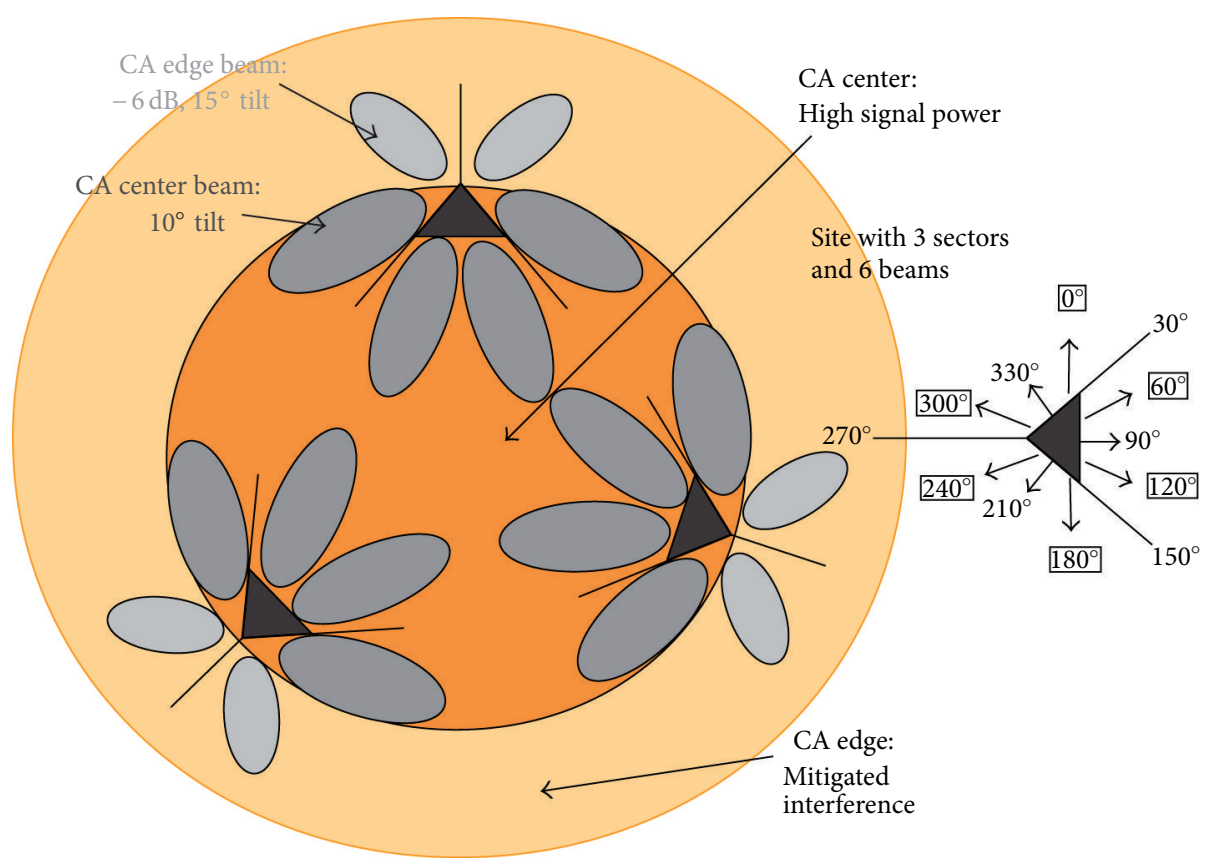

FIGURE 1: The complete interference mitigation framework consists of three adjacent sites. Multiple antennas at each of the cooperating BSs perform wideband beamforming to steer the signal more towards the CA's center.

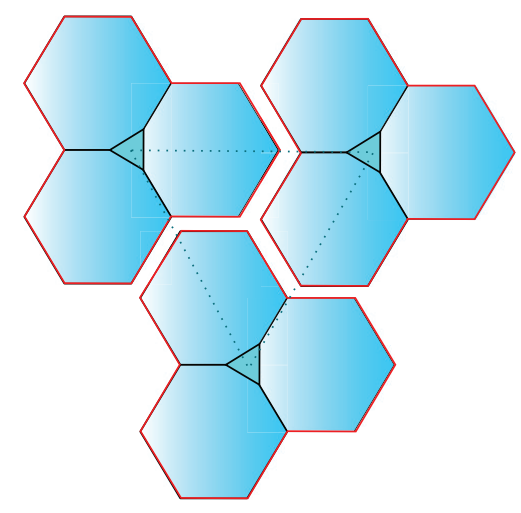

FIGURE 2: A CA consists of three adjacent sites.

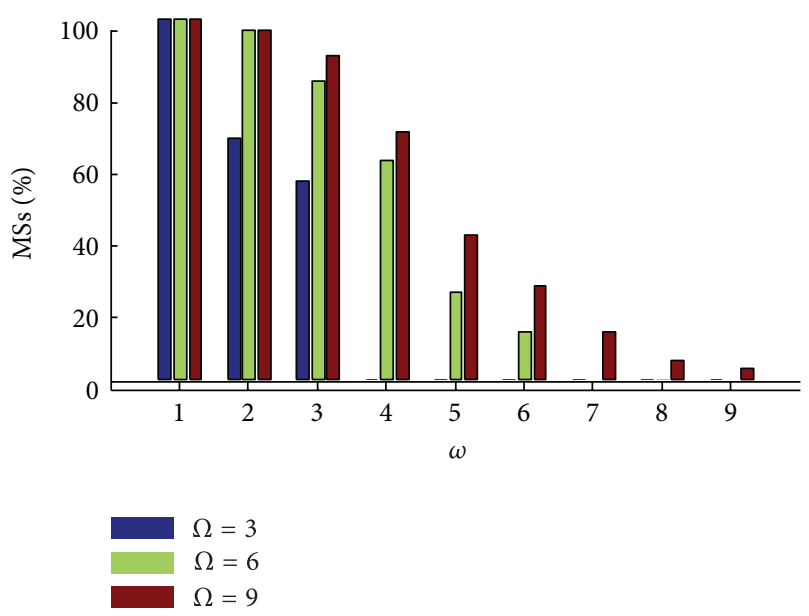

Figure 3: Penetration rate of user centric served MSs.
Regarding the channel matrix, the effective channels resulting from the wideband beams are used instead of the physical antenna coefficients. Thus, the number of columns in a channel matrix between a CA and its served MSs is equal to the number of wideband beamformers used per BS and the wideband beams are referred to as virtual antenna elements (vAEs). In order to be specific, the term "physical antenna elements (pAEs)" instead of AEs is used from now on.

Only vAEs are used for joint precoding. The corresponding matrix is denoted by $\widetilde{\mathbf{V}}$. Thus, each antenna weight in $\widetilde{\mathbf{V}}$ must be applied in the same manner at all pAEs, the corresponding vAE consists of.

Assuming a uniform linear array with a $\lambda / 2$ antenna spacing $(\lambda=$ wavelength), 4 pAEs at each BS and using $\sin (30 \mathrm{deg})=0.5$, the (normalized) beamforming vectors for each BS, directed to the left and right are given by $\vec{b}_{L}=$ $(1 / 4)\left[\begin{array}{llll}1 & j & -1 & -j\end{array}\right]^{T}$ and $\vec{b}_{R}=(1 / 4)\left[\begin{array}{llll}1 & -j & -1 & j\end{array}\right]^{T}$, respectively.

According to Figure 1 the wideband beams should also have an individual down tilt. Different down tilts can be realized if each single $\mathrm{pAE}$ of the uniform linear antenna arrays is extended to a vertical antenna array, again with, for example, $\lambda / 2$ spacing. The influences of the vertical antenna array-basically describing a so-called "active antenna"-can be included easily into the mathematical model if all rows of the (now) two-dimensional antenna array are concatenated to one long row which leads to increased lengths of $\vec{b}_{R}, \vec{b}_{L}$. Elements corresponding to different rows of the antenna array must have an additional phase offset according to the down tilt of center and edge beams. Furthermore, Tx power adaptation for cell edge beams is included by $a-6 \mathrm{~dB}$ powerdown-scaling of the corresponding beamforming vectors. In 

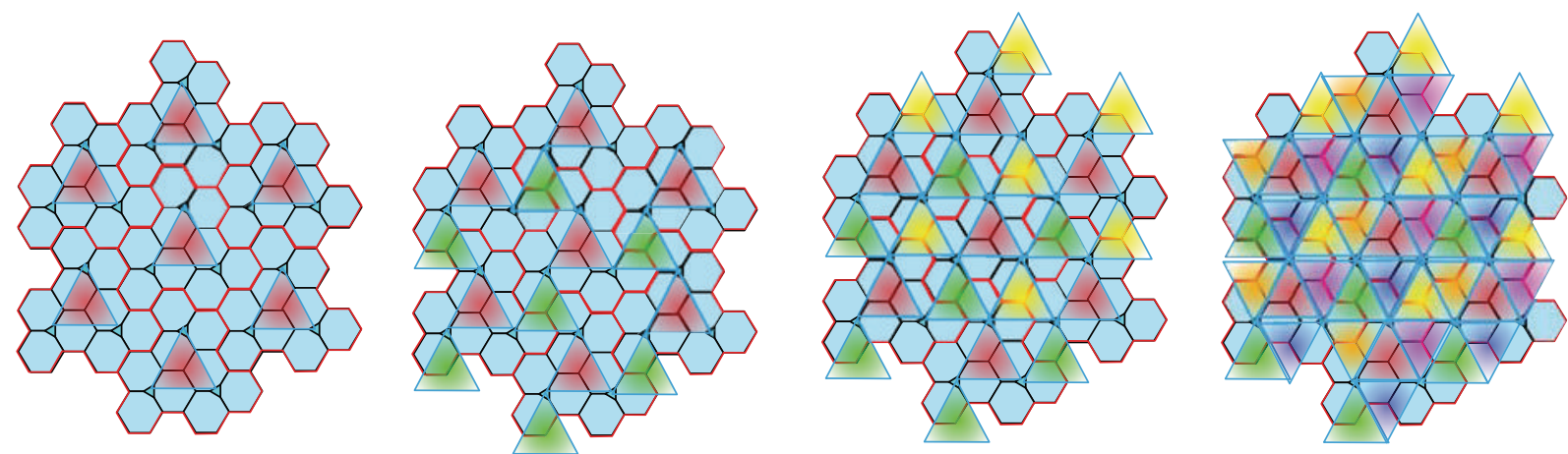

FIGURE 4: The basic clustering is illustrated on the most left picture. From left to the right, more and more cover shifts are added.

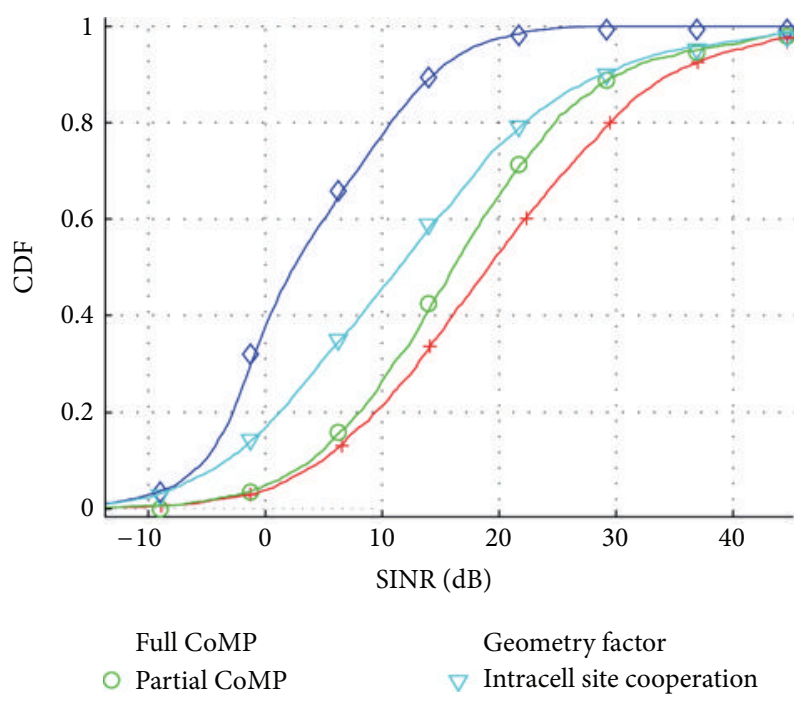

Figure 5: The partial CoMP concepts have a significant gain compared to intrasite cooperation scenarios, especially in the lowSINR regions.

total, four different extended beams result from different down tilts and Tx power reduction, denoted by ${ }_{c} \vec{b}_{R}, \vec{b}_{L}$ (center beams) and $\vec{b}_{R}, \vec{b}_{L}$ (edge beams). Figure 12 shows the positions of all these wideband beamformers. To keep the notation clear, the length extension of wideband beamformers is suppressed in the following since it has no relevant influence on the mathematical model. Furthermore, the down tilt could also be realized by two different pAEs with different down tilts, but would increase the hardware complexity.

In Figure 12, the first 6 wideband beams of a CA are depicted and numbered. The corresponding beamforming vectors are concatenated to a block diagonal matrix:

$$
\begin{aligned}
\widetilde{\mathbf{v}} & =\left[\widetilde{\mathbf{v}}_{1} \cdots \widetilde{\mathbf{v}}_{6}\right] \\
& =\left(\begin{array}{cc}
{\left[\vec{e}^{\vec{b}_{R}} \mid{ }_{c} \vec{b}_{L}\right]} & 0 \\
0 & {\left[\vec{c}_{R} \mid \vec{b}_{L}\right]} \\
& {\left[\vec{c}_{c} \mid \vec{b}_{L}\right]}
\end{array}\right) .
\end{aligned}
$$

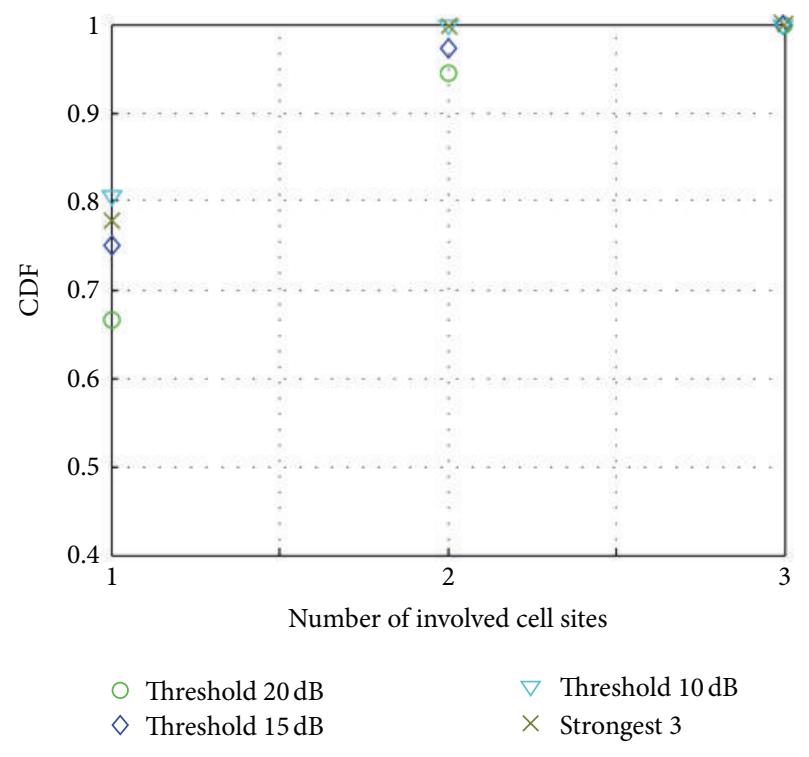

FIGURE 6: The number of involved sites in the set of reported BS depends on the number of reported BSs and thus on the threshold if that selection approach is chosen.

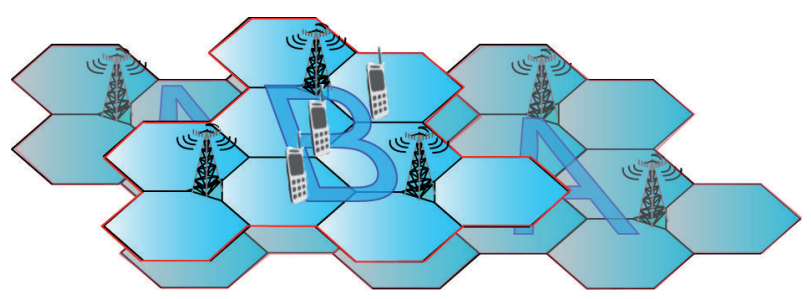

FIgURE 7: Using overlapping CAs, so-called "cover shifts" can avoid CA edge MSs.

The complete MIMO signal model can be described by

$$
\mathbf{y}=\mathbf{H} \widetilde{\mathbf{V}} \mathbf{V}=\widetilde{\mathbf{H}} \mathbf{V} .
$$

The central joint processing (CJP) calculates the joint precoding matrix $\mathbf{V}$ based on a channel matrix that is a result of the product between two matrices, that is, $\mathbf{H}$ and $\mathbf{V}$. Thus, $\mathbf{H}$ is 


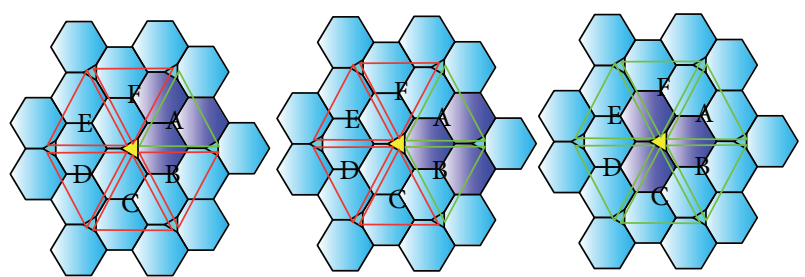

FIGURE 8: Dark hexagons denote the three network wide strongest BSs. Depending on their distribution, an MS can be scheduled into one, two, or six different cover shifts.

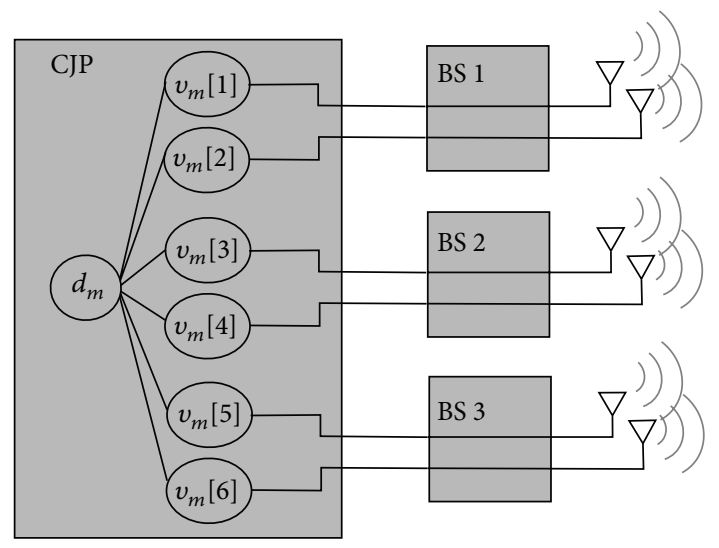

FIGURE 9: Scheme of cooperation between 3 BSs with 2 pAEs per BS. To do the central joint processing, a central unit calculates the joint precoding weights $\mathbf{v}_{m}[1], \ldots, \mathbf{v}_{m}[6]$ to transmit the symbol $d_{m}$ for MS $m$.

changed by $\widetilde{\mathbf{V}}$. These changes can also decrease the correlation between the individual elements in $\widetilde{\mathbf{H}}$ compared to $\mathbf{H}$. In other words the rank of a matrix consisting of vAEs might be higher compared to the case when the original pAEs are used as matrix elements. This improvement can occur in particular if $\widetilde{\mathbf{V}}$ is of reduced size.

Figure 10 depicts a possible realization of virtual AEs: The virtual antenna elements with tildes are to be applied on top of the physical antenna weights on the BSs by physical beamformers. (Here:, three BSs are used).

Figure 11 illustrates the results comparing SINR values utilizing the WB beams with and without CA-specific down tilt and power adaptation. In the latter case, a lower SINR can be observed compared to the CoMP simulations presented earlier in this article. Results presented in $[32,33]$ indicate that this effect comes from an increased inter-CA interference because the pathloss is not as dominant here compared to the CoMP reference scenario without any WB-beamforming.

If the WB-beam down tilt and power adaptation are activated, the results in Figure 11 illustrate the effect of interference floor shaping. Compared to the CoMP reference, a significant SINR gain can be observed, confirming the performance of the proposed interference shaping concept.

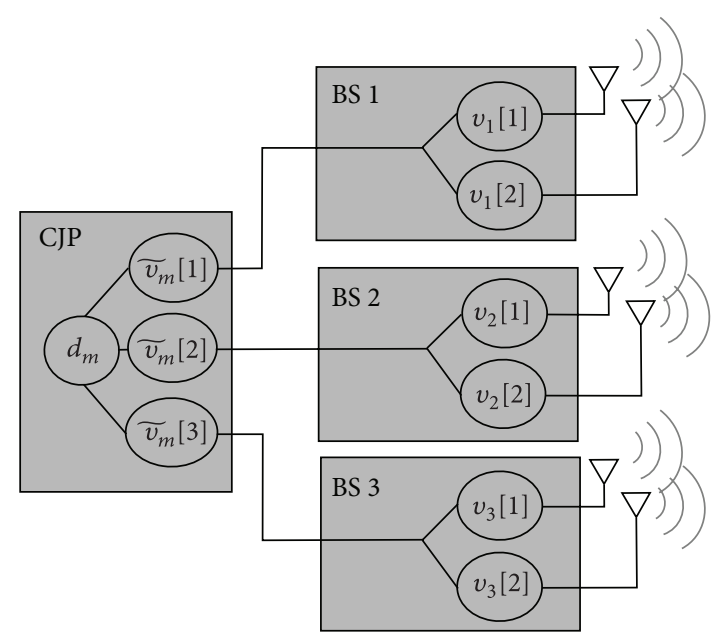

FIGURE 10: Each element of $\widetilde{\mathbf{v}}_{m}$ must be spread to all pAEs locally mounted at the BSs. Thus, there is much less cooperation overhead on the backhaul.

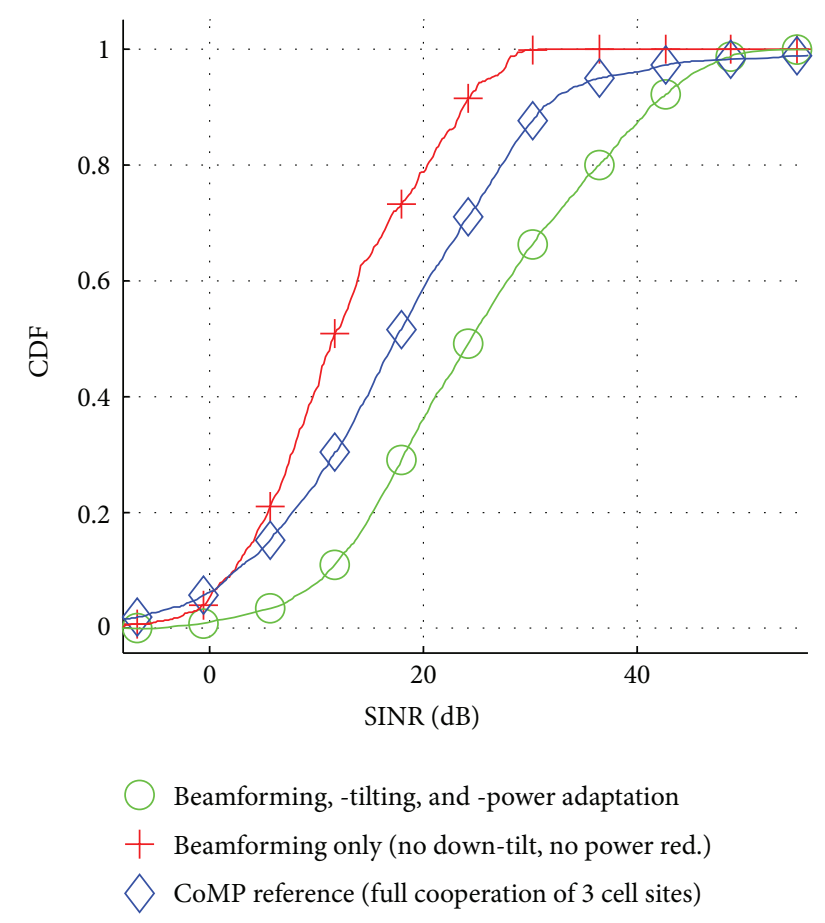

FIgURE 11: The blue line (diamonds) is the reference scenario as in Figure 5. Red (crosses) denotes the wide band (WB) beamforming switched on, but without any additional downtilt or power adaptation. Green (circles) contains the results for additional downtilt and power adaptation.

\section{Real World Issues}

The accuracy of the simulation results that were presented in the previous section is limited by the accuracy of the channel models that have been applied. However, the 3GPP 3D antenna model does not accurately describe the vertical dimension of a cellular system since it is constantly considered to have LOS characteristics. A more solid modelling 


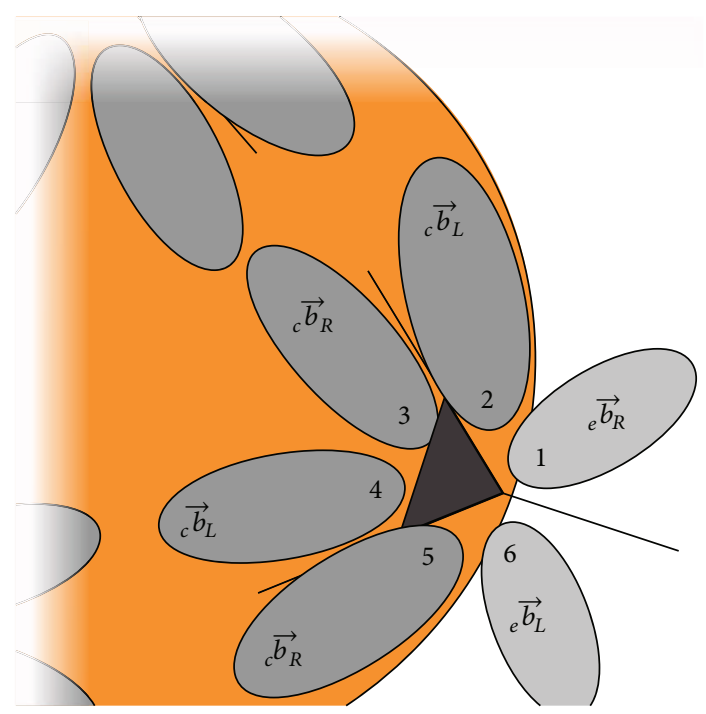

FIGURE 12: Numbering scheme for wideband beams at first site in a CA.

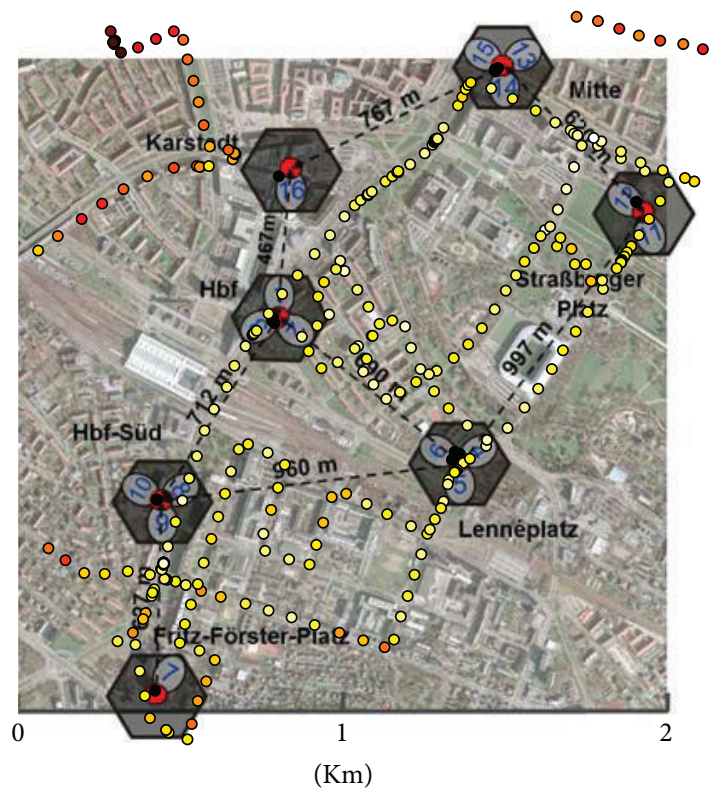

FIGURE 13: Field trial setup and measurement locations (colored dots) for the first field trial. The color indicates the SNR to the strongest base station for a downtilt of $6^{\circ}$.

would require the consideration of multiple reflection clusters as considered in the horizontal dimension. In order to validate the assumptions and results of the previous sections, we, thus, used extensive large scale field trials with regard to these most critical aspects. As depicted in Figure 13, the testbed consists of a total of 16 base stations that are deployed at seven sites. It covers a large part of the down town of the German city Dresden, an area with a typical urban building morphology that is characterized by large apartments of $20-$ $50 \mathrm{~m}$ height, a soccer stadium, a train station, railway tracts,

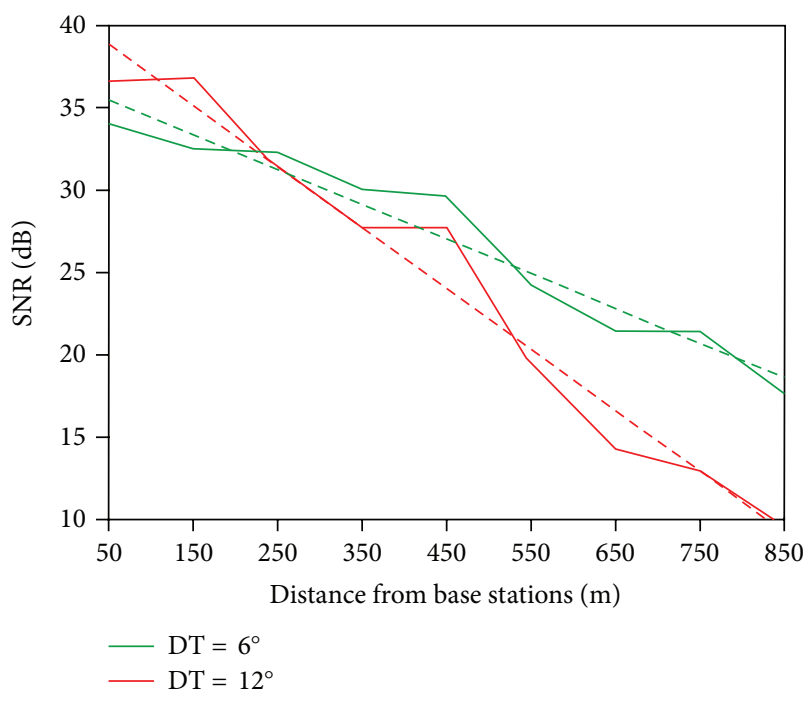

FIgURE 14: Average SNR (dB) over distance from base stations. Average over results at $16 \mathrm{BSs}$. The dashed lines show a linear fit.

and roads with up to 4 lanes. Four base stations are positioned in about $30 \mathrm{~m}$ and 12 base stations in about $50 \mathrm{~m}$ above ground, each equipped with a cross-polarized antenna (58 degrees half-power beamwidth and $14 \mathrm{dBi}$ gain). The intersite distance ranges between 500 and $1000 \mathrm{~m}$.

In a first field trial, we were interested in the impact of the downtilt on pathloss and coverage. In order to investigate this aspect, the same measurement route was traversed twice. For the first trial a downtilt of $6^{\circ}$ and for the second trial a downtilt of $12^{\circ}$ were used. During the trial a single UE transmitted OFDM pilots with transmit power of $37 \mathrm{dBm}$ over a bandwidth of $5.4 \mathrm{MHz}$ at a carrier frequency of $2.6 \mathrm{GHz}$. The received signal at all base stations was synchronously captured every $10 \mathrm{~s}$ and evaluated for channel and SNR estimation. For further details on the evaluation procedure, we refer the reader to $[21,34]$. The measurement locations are marked in Figure 13 as well, using coloured dots that indicate the SNR to the strongest base station for each location. In total 479 measurements were taken in each trial. For technical reasons, the measurement locations for both trials are not exactly the same which, however, does not effect the following results since the data is evaluated statistically. In Figure 14, we show how the average SNR depends on the distance to the base stations for the two different downtilt settings.

In order to evaluate the relationship between SNR and distance to the base station, we determined the distance to all 16 base stations for each measurement location. Figure 14 shows the average SNR that was measured at different distances to the base stations. However, for each location only the SNR of those base stations was considered which antennas were facing to the measurement location, with a tolerance of $30^{\circ}$. Figure 14 also shows that the SNR in $\mathrm{dB}$ decreases linearly with distance with an average SNR degradation (pathloss increase) of $2.1 \mathrm{~dB}$ and $3.7 \mathrm{~dB}$ every $100 \mathrm{~m}$ and for a downtilt of $6^{\circ}$ and $12^{\circ}$, respectively. The height of the base stations has a 
rather small effect on the average pathloss in our field trial. The results are useful to validate the results of our system level simulations and they show that an appropriate setting of the downtilts in a cover shift allows for separation of cooperation areas in a way that BSs that are facing outside the cooperation areas should apply a larger downtilt in order to decrease the interference to other cooperation areas. As described previously, coverage holes that would occur due to this strong isolation of cooperation areas would be closed by using different cover shifts on several layers that are separated by using different resources for each layer. Layer specific cover shifts could be realized by vertical beamforming. Our goal in the field trial that is described in the following was to explore how well different cooperation areas could be separated using these techniques. Since vertical beamforming was not available in the employed field trial system, we concentrated on the separation of a single cooperation area from neighboring base stations. The general field trial setup is the same as shown in Figure 14. The cooperation area of interest was made up of the three sites Hbf-Süd, Hbf, and Lennéplatz. Thus, in order to achieve a good coverage and large cooperation gains inside of the cooperation area a rather low downtilt of $7^{\circ}$ was chosen at base station 2, 6, 8 that are facing inward. At all other base stations of the cooperation area a larger downtilt of $17^{\circ}$ was chosen in order to minimize the interference to other cooperation areas. The downtilt at all surrounding base stations was $17^{\circ}$ as well as those would be interferers from surrounding cooperation clusters that transmit on the same resources. Figure 15 shows the result of a field trial where a single transmitting UE traversed through a wide area of the testbed. The colour of the measurement locations indicates the maximum SNR measured at any base station of the cooperation cluster. A black dot is shown at measurement locations where the SNR to all base station in the cooperation cluster is below $0 \mathrm{~dB}$ or where a base station that is not part of the cooperation cluster has a higher SNR than all base stations in the cooperation cluster. Compared to the previous trial in Figure 13, the transmit power was reduced to $18 \mathrm{dBm}$, thus the SNR is clearly lower as it would be achievable in a cellular downlink with macrobase stations.

An inherent feature of the interference mitigation concept being proposed is joint transmission of base stations in a cooperation cluster. The statistics for the SNR that is instantaneously achieved at several base stations for all measurement locations in the cooperation area is shown in Figure 16. For example the blue curve shows the CDF of SNR of the strongest base station at each location. The other solid curves show the CDF of the minimum SNR of instantaneously achieved at several base stations. For example at $40 \%$ of the measurement locations the SNR was larger than $10 \mathrm{~dB}$ at two base stations of the cooperation cluster instantaneously. Considering that the transmit power at a typical base station would be about $30 \mathrm{~dB}$ higher as that used in this field trial shows that there would be definitely a strong benefit from cooperation. The dashed curves show the SNR CDF of the strongest interfering base station. Thus, a partial feedback (and precoding) scheme would typically use cooperation of no more than four base stations. In order to increase this number, the presented framework incorporates the option for

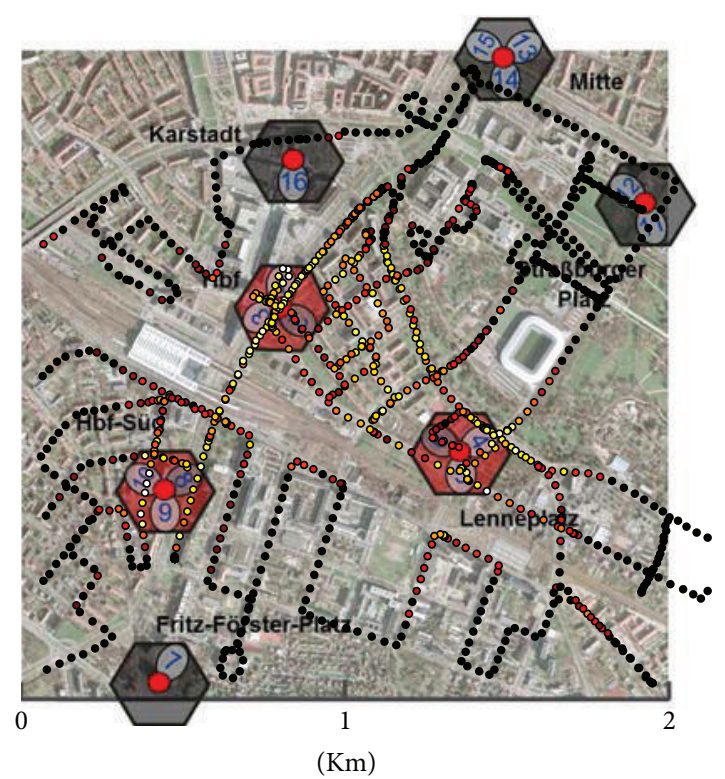

FIGURE 15: SNR in cooperation area using the tortoise concepts.

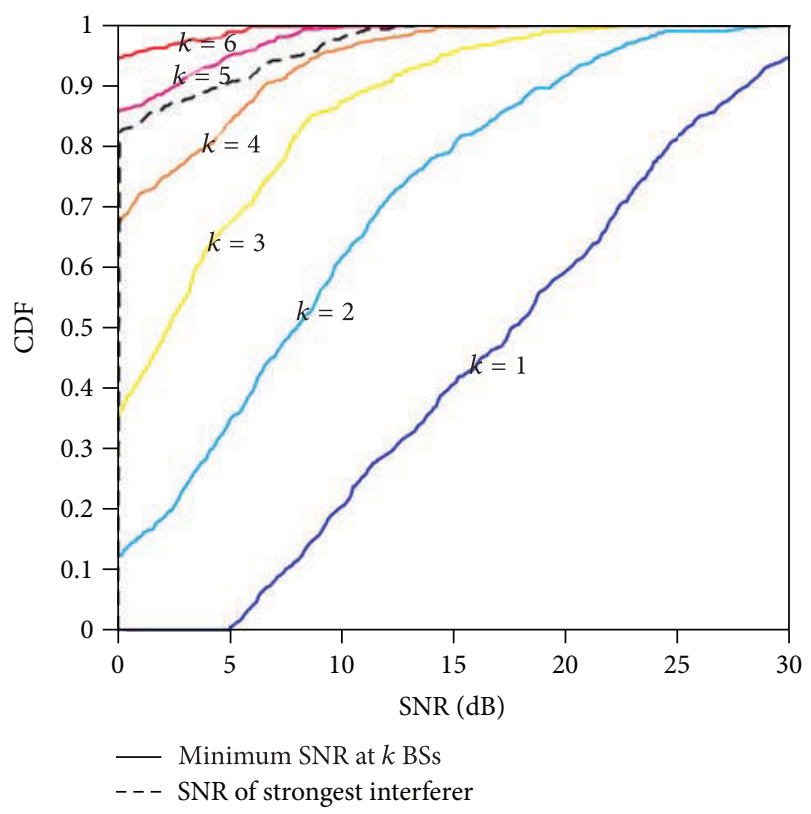

FIGURE 16: CDF of SNR at the base stations in the CA. The dashed curve shows the CDF of the SNR of the first interferer from outside of the CA. We see that typically 4 base stations in the CA have a higher SNR than the first interferer.

power control as presented in Section 4.6. Figure 17 shows the impact of power setting, where base stations that face out of the cooperation area have a transmit power that is reduced by $6 \mathrm{~dB}$. Since this field trial was done in the uplink, the impact of a reduced transmit power was obtained by a reduction of the measured SNR. While the reduced transmit power has only small effect on the SNR in the cooperation cluster, the strongest interferer is reduced by $6 \mathrm{~dB}$ resulting in a significant increase of the average SINR. 


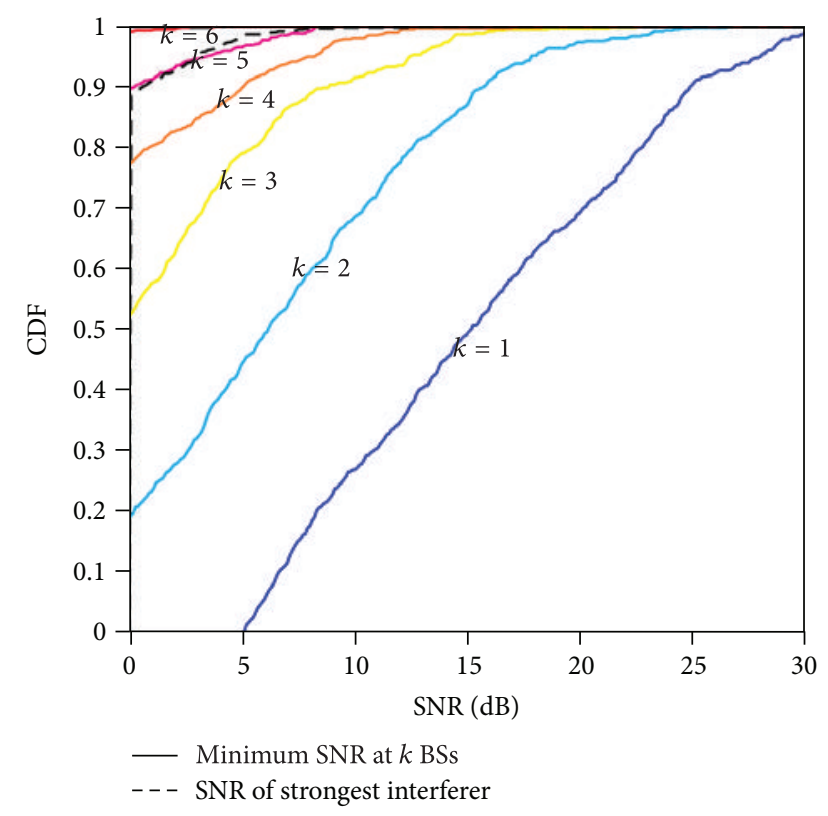

FIgURE 17: The same as Figure 15 but with power control.

\section{Cooperative Scheduling Strategies-Converting SINR into Spectral Efficiency}

Applying the above described framework increases the SINR per UE significantly for the single UE per cell scenario as obvious from Figure 11. This is a big step into the direction of noticeable system level performance gains, but leaves a main topic unsolved, that is, the optimum multiuser CoMP scheduling.

CoMP scheduling is - similar as for MU-MIMO-a challenge, requiring ideally an exhaustive search in all potential scheduling and precoding dimensions. The goal is to maximize the spectral efficiency while keeping a predefined degree of fairness or even better improving the degree of fairness. This is equivalent to maximizing the constrained sum rate over all simultaneously served data streams per CA, where the constraint depends on the intended degree of fairness.

For JP CoMP the search space generally comprise the following search dimensions.

(i) Setup of CAs with the goal to serve as many as possible MSs from their most appropriate cells, as being explained above.

(ii) In case of overlapping clusters according to the cover shift concept the scheduler has to consider different allocations of MSs to cover shift.

(iii) The grouping of simultaneously served MSs per CA is a very important step for finding the mutually most orthogonal set of users for a certain subcarrier or PRB. Independent of the specifically used linear precoding technique, like optimum Wiener filtering, zero forcing, signal to noise and leakage minimization, and so forth the PNL $\alpha$ as being introduced in (4) will explode for a badly conditioned channel matrix $\mathbf{H}$.

(iv) User grouping is closely related with determining the optimum number of data streams per CA, that is how many users are served with how many data streams. Generally there is a tradeoff. With increasing number of data streams the sum rate increases up to the point, where the precoding normalization loss $\alpha$ overcomes the multistream throughput gains. $\alpha$ combines a splitting loss due the partitioning of the Tx power to more streams and the loss for orthogonalization of the data streams.

(v) For each user group and rank the optimum $T x$ - and $R x$ - beamformers for the BS and MSs have to be derived.

(vi) As one of the last steps frequency dependent scheduling of MS sets to PRBs or subcarriers has to maximize overall capacity, while ensuring at least proportional fairness for all MSs. Taking all these 6 dimensions into account, one can formulate the overall optimization which must be done comprising
(a) cover shifts,
(b) user groups,
(c) precoder,
(d) postcoder,
(e) number of streams, and
(f) PRBs.

Obviously, the overall search space easily explodes even for small number of MSs requiring a powerful heuristic scheduling strategy.

A completely new score based scheduler has been implemented taking advantage of the concepts presented in last sections, that is, being limited to one single CA. The description of all applied scheduling strategies is beyond the scope of this paper, but so far achieved results look very promising. It was possible to serve $3 \mathrm{MSs}$ per cell with reasonable data rates, leading to a mean throughput of about $7 \mathrm{bit} / \mathrm{s} / \mathrm{Hz} / \mathrm{cell}$ for an urban macro $4 \times 2$ MIMO scenario. This is more than a factor of 2 larger than what has been reported in 3GPP for the same scenario as outcome of the CoMP study item (3.1 bits/s/Hz/cell).

\section{Conclusion}

Interference mitigation in cellular mobile radio systems is a challenging problem for the high number of interferers that are potentially wide spread between far off BSs. Network wide cooperation is well known to be extremely powerful in theory, but practical feasible systems have to divide the network into smaller cooperation areas (i.e., clusters). As a consequence there will be inter cooperation area interference, spoiling most of the gains observed for network wide interference free transmission. To get back to similar performance as for network wide cooperation we propose a framework for interference mitigation, including oversized clusters and 
overlapping cooperation areas, interference floor shaping by means of wideband beamformers, and a novel cooperative score based scheduler. The performance of all that concepts has been investigated by a software simulation framework written in Matlab and by real world field trials made at the TU-Dresden-LTE-Testbed.

The results indicate that the ideas can really be applied in future networks with significant gains regarding the SIR/ SINR. For the future, further fine tuning is planned to reach even higher gains.

\section{Abbreviations}

$\begin{array}{ll}\text { 3GPP: } & \text { 3rd generation partnership project } \\ \text { AE: } & \text { Antenna element } \\ \text { BS: } & \text { Cell } \\ \text { CA: } & \text { Cooperation area } \\ \text { CDMA: } & \text { Code division multiple access } \\ \text { CJP: } & \text { Central joint processing } \\ \text { CoMP: } & \text { Cooperative multi point transmission } \\ \text { CSI: } & \text { Channel state information } \\ \text { CU: } & \text { Central unit } \\ \text { FFP: } & \text { Fractional frequency partitioning } \\ \text { ICI: } & \text { Inter cell interference } \\ \text { ICIC: } & \text { Inter cell interference coordination } \\ \text { IRC: } & \text { Interference rejection combining } \\ \text { ISD: } & \text { Inter site distance } \\ \text { JP: } & \text { Joint precoding } \\ \text { JT: } & \text { Joint transmission } \\ \text { LOS: } & \text { Line of sight } \\ \text { LTE: } & \text { Long term evolution } \\ \text { MIMO: } & \text { Multiple input multiple output } \\ \text { MU-MIMO: } & \text { Multi user MIMO } \\ \text { MS: } & \text { Mobile station } \\ \text { NLOS: } & \text { Non line of sight } \\ \text { OFDMA: } & \text { Orthogonal frequency division multiple } \\ \text { pAE: } & \text { access } \\ \text { PCR: } & \text { Physical antenna element } \\ \text { PRB: } & \text { Partial channel reporting } \\ \text { PNL: } & \text { Physical resource block } \\ \text { SCME: } & \text { Precoding normalization loss } \\ \text { SINR: } & \text { Spatial channel model extended } \\ \text { SIR: } & \text { Signal to noise and interference ratio } \\ \text { SISO: } & \text { Signal to interference ratio } \\ \text { vAE: } & \text { Single input single outpout } \\ & \text { Virtual antenna element. } \\ & \end{array}$

\section{References}

[1] S. Redl, M. Weber, and M. Oliphant, GSM and Personal Communications Handbook, Artech House, 1998.

[2] K. Rawer, Wave Propagation in the Ionosphere, Kluwer Academic, 1993.

[3] H. Dai, A. F. Molisch, and H. V. Poor, "Downlink capacity of interference-limited MIMO systems with joint detection," IEEE Transactions on Wireless Communications, vol. 3, no. 2, pp. 442453, 2004.

[4] A. Sendonaris and V. Veeravalli, "The capacity-coverage tradeoff in CDMA systems with soft handoff," in Proceedings of the 31st Asilomar Conference on Signals, Systems \& Computers, vol. 1, pp. 625-629, IEEE, November 1997.

[5] R. Kwan, C. Leung, and J. Zhang, "Proportional fair multiuser scheduling in LTE," IEEE Signal Processing Letters, vol. 16, no. 6, pp. 461-464, 2009.

[6] D. Kimura, Y. Harada, and H. Seki, "De-centralized dynamic ICIC using X2 interfaces for downlink LTE systems," in Proceedings of the 73rd IEEE Vehicular Technology Conference (VTC '11-Spring), pp. 1-5, IEEE, May 2011.

[7] P. W. Baier, M. Meurer, T. Weber, and H. Troeger, "Joint transmission (JT), an alternative rationale for the downlink of time division CDMA using multi-element transmit antennas," in Proceedings of the 6th IEEE International Symposium on Spread Spectrum Techniques and Applications, vol. 1, pp. 1-5, IEEE, September 2000.

[8] N. Jindal, W. Rhee, S. Vishwanath, S. A. Jafar, and A. Goldsmith, "Sum power iterative water-filling for multi-antenna Gaussian broadcast channels," IEEE Transactions on Information Theory, vol. 51, no. 4, pp. 1570-1580, 2005.

[9] G. J. Foschini, K. Karakayali, and R. A. Valenzuela, "Coordinating multiple antenna cellular networks to achieve enormous spectral efficiency," IEE Proceedings, vol. 153, no. 4, pp. 548-555, 2006.

[10] M. K. Karakayali, G. J. Foschini, and R. A. Valenzuela, "Network coordination for spectrally efficient communications in cellular systems," IEEE Wireless Communications, vol. 13, no. 4, pp. 5661, 2006.

[11] C. Botella, T. Svensson, X. Xu, and H. Zhang, "On the performance of joint processing schemes over the cluster area," in Proceedings of the 71st IEEE Vehicular Technology Conference (VTC '10-Spring), pp. 1-5, IEEE, May 2010.

[12] P. Marsch and G. P. Fettweis, Coordinated Multi-Point in Mobile Communications: From Theory to Practice, Cambridge University Press, 2011.

[13] 3GPP, Coordinated Multi-Point Operation for LTE. TR 36.819, 3rd Generation Partnership Project (3GPP), 062011.

[14] A. Osseiran, J. F. Monserrat, and W. Mohr, Mobile and Wireless Communications for IMT-Advanced and Beyond, Wiley, 2011.

[15] D. S. Baum, J. Hansen, and J. Salo, "An interim channel model for beyond-3G systems: extending the 3GPP spatial channel model (SCM)," in Proceedings of the 61st IEEE Vehicular Technology Conference (VTC '05-Spring), vol. 5, pp. 3132-3136, IEEE, June 2005.

[16] N. U. Hassan, C. Yuen, and Z. Zhang, "Optimal power control between two opportunistic cooperative base stations," in Proceedings of the 13th IEEE International Workshop on Signal Processing Advances in Wireless Communications (SPAWC '12), pp. 194-198, June 2012.

[17] M. Eslamifar, W. H. Chin, C. Yuen, and G. Y. Liang, "Performance analysis of two-way multiple-antenna relaying with network coding," in Proceedings of the 70th IEEE Vehicular Technology Conference Fall (VTC '09-Fall), pp. 1-5, IEEE, September 2009.

[18] V. D’Amico, A. Dekorsy, A. Gouraud et al., "ARTIST4G a way forward to the interference problem in future mobile networks," in Proceedings of Future Network and Mobile Summit, pp. 1-8, IEEE, June 2010.

[19] W. Mennerich and W. Zirwas, "User centric coordinated multi point transmission," in Proceedings of the 72nd IEEE Vehicular Technology Conference (VTC '10-Fall), Ottawa, Canada, September 2010 . 
[20] W. Mennerich and W. Zirwas, "Implementation issues of the partial CoMP concept," in Proceedings of the 21st International Symposium on Personal Indoor and Mobile Radio Communications (PIMRC '10), pp. 1939-1944, Istanbul, Turkey, September 2010.

[21] M. Grieger, G. Fettweis, and P. Marsch, "Large scale field trial results on uplink CoMP with multi antenna base stations," in Proceedings of the IEEE Vehicular Technology Conference (VTC '11-Fall), pp. 1-5, September 2011.

[22] R. Hunger, F. A. Dietrich, M. Joham, and W. Utschick, "Robust transmit zero-forcing filters," in Proceedings of ITG Workshop on Smart Antennas, pp. 130-137, IEEE, March 2004.

[23] T. Yoo and A. Goldsmith, "On the optimality of multiantenna broadcast scheduling using zero-forcing beamforming," IEEE Journal on Selected Areas in Communications, vol. 24, no. 3, pp. 528-541, 2006.

[24] C. B. Peel, B. M. Hochwald, and A. L. Swindlehurst, "A vector-perturbation technique for near-capacity multiantenna multiuser communication-part I: channel inversion and regularization," IEEE Transactions on Communications, vol. 53, no. 1, pp. 195-202, 2005.

[25] B. M. Hochwald, C. B. Peel, and A. L. Swindlehurst, "A vectorperturbation technique for near-capacity multiantenna multiuser communication-part II: perturbation," IEEE Transactions on Communications, vol. 53, no. 3, pp. 537-544, 2005.

[26] M. H. M. Costa, "Writing on dirty paper," IEEE Transactions on Information Theory, vol. 29, no. 3, pp. 439-441, 1983.

[27] A. Goldsmith, S. A. Jafar, N. Jindal, and S. Vishwanath, "Capacity limits of MIMO channels," IEEE Journal on Selected Areas in Communications, vol. 21, no. 5, pp. 684-702, 2003.

[28] D. Gesbert, M. Shafi, D. S. Shiu, P. J. Smith, and A. Naguib, "From theory to practice: an overview of MIMO space-time coded wireless systems," IEEE Journal on Selected Areas in Communications, vol. 21, no. 3, pp. 281-302, 2003.

[29] W. Yu and T. Lan, "Transmitter optimization for the multiantenna downlink with per-antenna power constraints," IEEE Transactions on Signal Processing, vol. 55, no. 6, pp. 2646-2660, 2007.

[30] S. Kaviani and W. Krzymień, "Optimal multiuser zero forcing with per-antenna power constraints for network MIMO coordination," EURASIP Journal on Wireless Communications and Networking, vol. 2011, Article ID 190461, 2011.

[31] W. Mennerich and W. Zirwas, "User centric scheduling in cooperative networks," in Proceedings of the 1st Middle East Conference on Antennas and Propagation (MECAP '10), pp. 15, Cairo, Egypt, October 2010.

[32] W. Mennerich and W. Zirwas, "Interference floor shaping for cooperative cellular radio systems," in Proceedings of the 74th IEEE Vehicular Technology Conference (VTC '11-Fall), San Francisco, Calif, USA, September 2011.

[33] W. Mennerich and W. Zirwas, "Reporting effort for cooperative systems applying interference floor shaping," in Proceedings of 22nd Annual IEEE International Symposium on Personal, Indoor and Mobile Radio Communications (PIMRC '11), pp. 541-545, Toronto, Canada, September 2011.

[34] M. Grieger and G. P. Fettweis, "Impact of antenna downtilt on cooperative uplink detection in a large scale field trial," in Proceedings of GLOBECOM Workshops (GLOBECOM '11), pp. 431-435, Houston, Tex, USA, December 2011. 

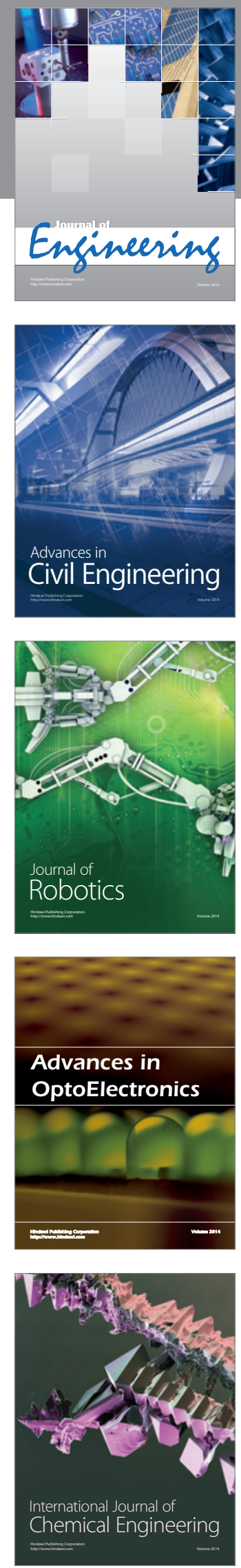

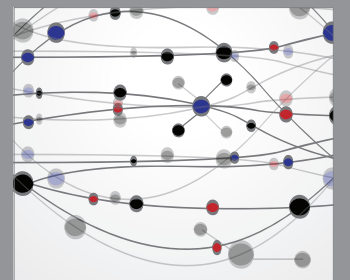

The Scientific World Journal
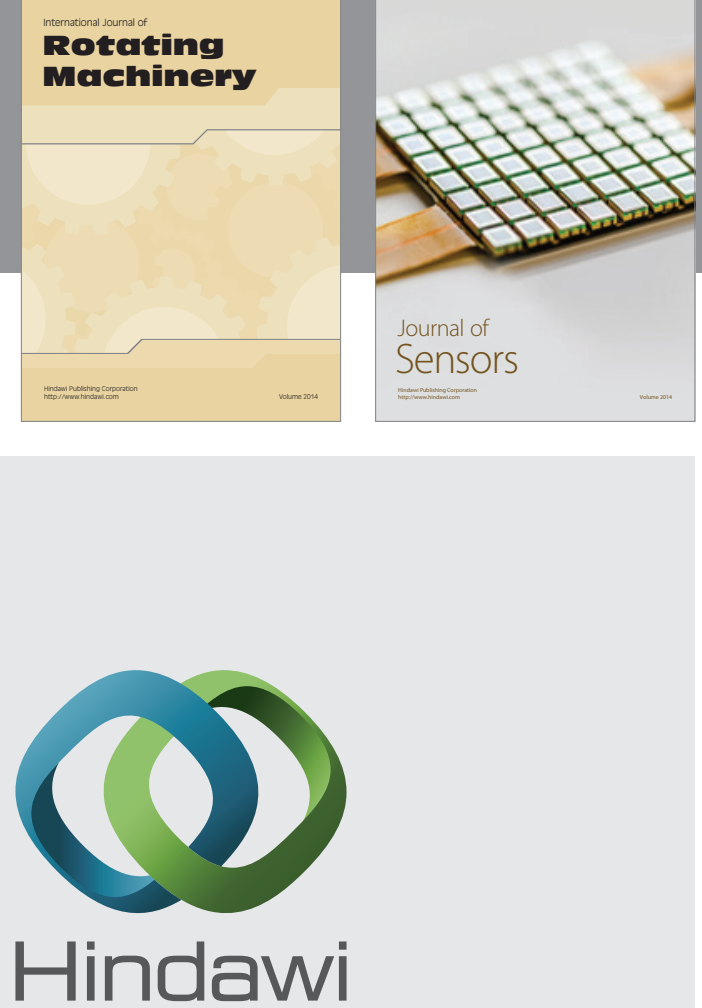

Submit your manuscripts at http://www.hindawi.com
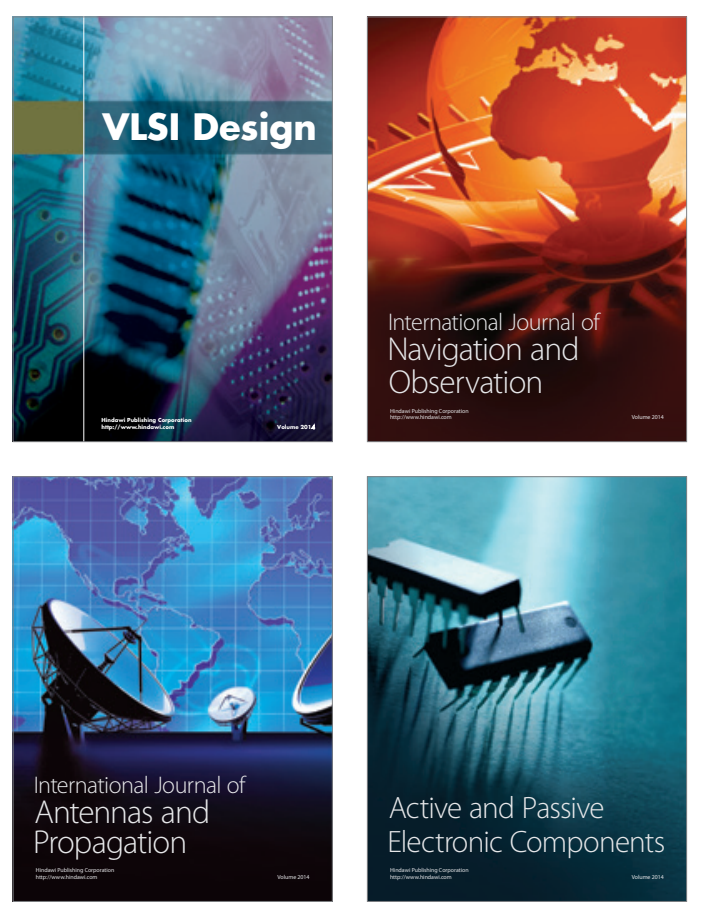
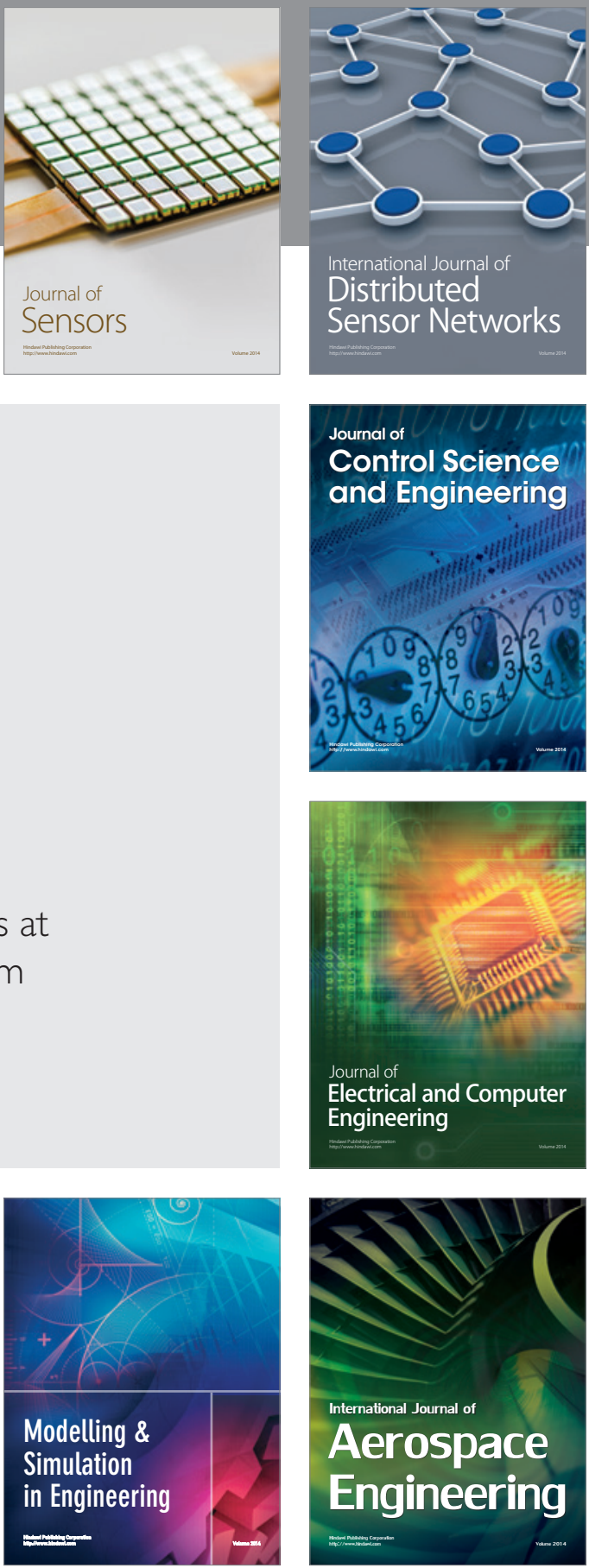

Journal of

Control Science

and Engineering
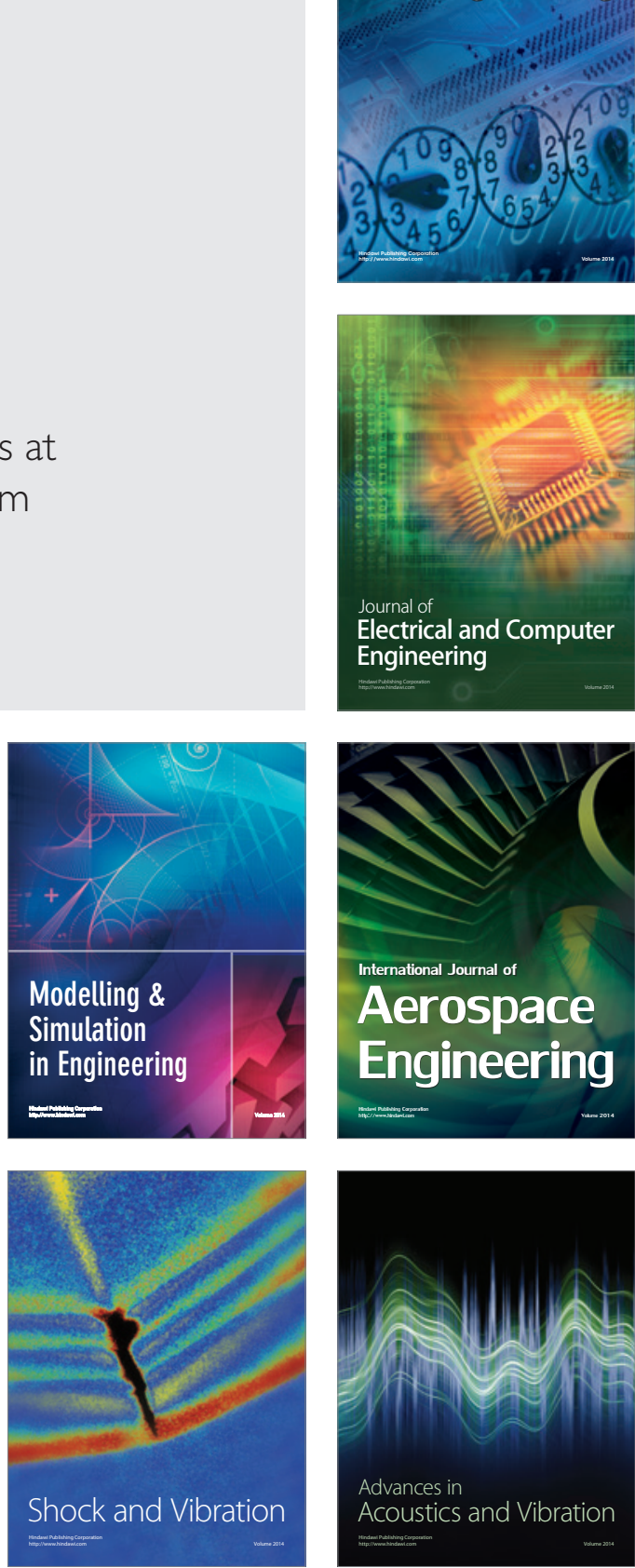